\title{
Control of induction motors: an adaptive passivity MIMO perspective
}

\author{
Manuel A. Duarte-Mermoud ${ }^{1, * \dagger}$ and Juan C. Travieso ${ }^{2, \$}$ \\ ${ }^{1}$ Electrical Engineering Department, University of Chile, Av. Tupper 2007, Casilla 412-3, Santiago, Chile \\ ${ }^{2}$ Electrical Engineering Department, University of Santiago of Chile, Av. Ecuador 3519, Casilla 10233, Santiago, Chile
}

\begin{abstract}
SUMMARY
The design of two multiple-input multiple-output (MIMO) controllers for induction motors, based on adaptive passivity, is presented in this paper. The controller design method is based on concepts of equivalence passivity via adaptive feedback, previously developed by the authors. Robustness under variations of the motor-load parameters is guaranteed and the knowledge of such a parameters is not needed in the design. Simple proportional controllers for the torque, rotor flux and stator current control loops are used, due to the control simplification introduced by the use of feedback passive equivalence. A principle called 'Torque-Flux Control Principle' is used in this article introducing a considerable simplification in the resultant controller. Because of the employment of this principle, the control efforts are diminished and rotor flux estimation (or measurement) is avoided. Copyright (C) 2003 John Wiley \& Sons, Ltd.
\end{abstract}

\section{INTRODUCTION}

The design of suitable non-linear control algorithms for induction motor has been widely investigated during this decade. The speed, torque and rotor flux controllers continue evolving based now on techniques such as sliding modes and passivity [1-5]. All the control schemes that guarantee high performance control uses also the field orientation principle [6-9].

Due to their own characteristics non-linear techniques do guarantee a suitable machine operation for all the specified range and they also consider parameter variations of the set motor-load. Sliding mode techniques [1-3, 10-12] guarantee robustness under some parameter variations such as load torque and rotor resistance. Nevertheless this control technique presents the chattering effect and acoustic noise as main disadvantages. On the other hand, passivitybased controllers $[3,4]$ are able to simplify the control. However, all this techniques are based on complex control schemes that involve on line parameter estimation at every instant of time, state variable estimation (rotor flux estimation) and control strategy based on these estimations [13-16]. For example, we can cite different type of control techniques used to face this problem,

\footnotetext{
*Correspondence to: Dr. Manuel A. Duarte-Mermoud, Electrical Engineering Department, University of Chile, Av. Tupper 2007, Casilla 412-3, Santiago, Chile

†E-mail: mduartem@cec.uchile.cl

†E-mail: jtravies@lauca.usach.cl
}

Contract/grant sponsor: CONICYT-CHILE; contract/grant number: 1000937

Copyright (C) 2003 John Wiley \& Sons, Ltd.

Received 27 June 2002

Revised 7 November 2002

Accepted 6 February 2003 
such as robust discrete-time stabilization [17], nonlinear robust output feedback [18,19], state space $H_{\infty}$ [20], non-linear predictive control [21] and PI control [22].

In Reference [5] the design of two single-input single-output (SISO) controllers for induction motors based on adaptive passivity was presented [23,24]. These strategies were suitably simplified by using the new principle called 'Torque-Flux Control Principle' which is also presented in this paper in Section 4. The design of two controllers for induction motors also based on adaptive passivity is presented in this paper, but from a multiple-input multiple-output (MIMO) perspective [25,26]. These control strategies are described in Section 3 and simplified using the principle called 'Torque-Flux Control Principle' in Section 4, obtaining two control schemes without requiring parameter and variable estimations and using simple proportional gains for the speed, rotor flux and stator currents control loops. One scheme uses fixed adaptive gains and the other employs time-varying adaptive gains. Both controllers present only two matrix adjustable parameters by means of simple adaptive laws, guaranteeing stability and robustness under a wide range of motor-load parameter variations, as well as under a wide range of proportional gain variations. The proposed controllers here give a similar performance than those presented in Reference [5] using a SIS perspective, as can be seen in Section 5.

\section{MOTOR MODEL AND PASSIVE DECOMPOSITION SCHEME}

\subsection{Induction motor model}

An induction motor model, obtained from the generalized electrical machine equations, will be used in this study, with arbitrary reference system of $x-y$ co-ordinates rotating at a generic speed $\omega_{\mathrm{g}}$. Magnetic field linearly distributed through the air gap is assumed and the resulting model is in general applicable to a p-poles machine. Iron losses, saturation and hysteresis phenomena are neglected. The resulting equations are [27]

$$
\begin{aligned}
& \dot{i}_{\mathrm{sx}}=-\left(\frac{R_{\mathrm{s}}}{\sigma L_{\mathrm{s}}}+\frac{L_{\mathrm{m}}^{2} R_{\mathrm{r}}}{\sigma L_{\mathrm{s}} L_{\mathrm{r}}^{2}}\right) i_{\mathrm{sx}}+\omega_{\mathrm{g}} i_{\mathrm{sy}}+\frac{L_{\mathrm{m}}}{\sigma L_{\mathrm{s}} L_{\mathrm{r}}^{2}} R_{\mathrm{r}} \psi_{\mathrm{rx}}+\frac{L_{\mathrm{m}}}{\sigma L_{\mathrm{s}} L_{\mathrm{r}}} \omega_{\mathrm{r}} \psi_{\mathrm{ry}}+\frac{1}{\sigma L_{\mathrm{s}}} u_{\mathrm{sx}} \\
& \dot{i}_{\mathrm{sy}}=-\omega_{\mathrm{g}} i_{\mathrm{sx}}-\left(\frac{R_{\mathrm{s}}}{\sigma L_{\mathrm{s}}}+\frac{L_{\mathrm{m}}^{2} R_{\mathrm{r}}}{\sigma L_{\mathrm{s}} L_{\mathrm{r}}^{2}}\right) i_{\mathrm{sy}}-\frac{L_{\mathrm{m}}}{\sigma L_{\mathrm{s}} L_{\mathrm{r}}} \omega_{\mathrm{r}} \psi_{\mathrm{rx}}+\frac{L_{\mathrm{m}} R_{\mathrm{r}}}{\sigma L_{\mathrm{s}} L_{\mathrm{r}}^{2}} \psi_{\mathrm{ry}}+\frac{1}{\sigma L_{\mathrm{s}}} u_{\mathrm{sy}} \\
& \dot{\psi}_{\mathrm{rx}}=R_{\mathrm{r}} \frac{L_{\mathrm{m}}}{L_{\mathrm{r}}} i_{\mathrm{sx}}-\frac{R_{r}}{L_{r}} \psi_{\mathrm{rx}}+\left(\omega_{\mathrm{g}}-\omega_{\mathrm{r})} \psi_{\mathrm{ry}}\right. \\
& \dot{\psi}_{\mathrm{ry}}=R_{\mathrm{r}} \frac{L_{\mathrm{m}}}{L_{\mathrm{r}}} i_{\mathrm{sy}}-\left(\omega_{\mathrm{g}}-\omega_{\mathrm{r})} \Psi_{\mathrm{rx}}-\frac{R_{\mathrm{r}}}{L_{\mathrm{r}}} \psi_{\mathrm{ry}}\right. \\
& \dot{\omega}_{\mathrm{r}}=\left(T_{\mathrm{em}}-T_{\mathrm{c}}\right) \frac{1}{J}-\frac{B_{\mathrm{p}}}{J} \omega_{\mathrm{r}}
\end{aligned}
$$

where $i_{\mathrm{sx}}, i_{\mathrm{sy}}$ are the stator currents, $\psi_{\mathrm{rx}}, \psi_{\mathrm{ry}}$ are the rotor fluxes, $\omega_{\mathrm{r}}$ is the rotor speed and $u_{\mathrm{sx}}, u_{\mathrm{sy}}$ are the stator voltages, considered as control inputs. $L_{\mathrm{m}}, L_{\mathrm{s}}, L_{\mathrm{r}}$ are the mutual, stator and rotor inductances, respectively. $R_{\mathrm{S}}, R_{\mathrm{r}}$ are the stator and rotor resistances, respectively. $J$ is the rotor inertia, $T_{\mathrm{em}}$ is the electromagnetic torque produced by the motor, $T_{\mathrm{c}}$ is the load torque and $B_{\mathrm{p}}$ is the mechanical viscous damping coefficient.

We also define $\sigma=1-L_{\mathrm{m}}^{2} / L_{\mathrm{s}} L_{\mathrm{r}}$ as he leakage or coupling factor, $R_{\mathrm{s}}^{\prime}=R_{\mathrm{s}}+L_{\mathrm{m}}^{2} / L_{\mathrm{r}}^{2} R_{\mathrm{r}}$ as the stator transient resistance and $\sigma L_{\mathrm{s}}$ as the stator transient inductance. 
Furthermore, the electromagnetic torque is given by

$$
T_{\mathrm{em}}=\frac{3 p L_{\mathrm{m}}}{22 L_{\mathrm{r}}}\left(\psi_{\mathrm{rx}} i_{\mathrm{sy}}-\psi_{\mathrm{ry}} i_{\mathrm{sy}}\right)
$$

\subsection{Passive decomposition}

A passive decomposition valid also for the generalized machine is used in Reference [4]. It considers the induction motor as the interconnection of the electrical and mechanical systems by means of a negative feedback, as seen in Figure 1. Starting from the previous statement the induction motor control can be carried out by controlling the electrical subsystem, considering the mechanical subsystem as a passive disturbance $\left(T_{\mathrm{em}}-T_{\mathrm{c}}\right)$ on the variable $\omega_{\mathrm{r}}$.

Starting from the previous scheme, we define the variable errors as the difference between the desired variable and the real one. For example, $e_{i_{\mathrm{sx}}}=i_{\mathrm{sx}}^{*}-i_{\mathrm{sx}}$, where $i_{\mathrm{sx}}^{*}$ is the reference of the direct current component and $i_{\mathrm{SX}}$ the direct current component obtained through measurements. Therefore, we define all the variable errors as follows:

$$
\begin{gathered}
e_{i_{\mathrm{sx}}}(t)=i_{\mathrm{sx}}^{*}(t)-i_{\mathrm{sx}}(t), \quad e_{i_{\mathrm{sy}}}(t)=i_{\mathrm{sy}}^{*}(t)-i_{\mathrm{sy}}(t) \\
e_{\psi_{\mathrm{rx}}}(t)=\psi_{\mathrm{rx}}^{*}(t)-\psi_{\mathrm{rx}}(t), \quad e_{\psi_{\mathrm{ry}}}(t)=\psi_{\mathrm{ry}}^{*}(t)-\psi_{\mathrm{ry}}(t)
\end{gathered}
$$

Then we can write the electrical subsystem in term of the variable errors as follows:

$$
\begin{gathered}
\dot{y}=\left[\begin{array}{c}
\dot{e}_{i_{\mathrm{sx}}} \\
\dot{e}_{i_{\mathrm{sy}}}
\end{array}\right]=\left[\begin{array}{c}
-\frac{R_{\mathrm{s}}^{\prime}}{\sigma L_{\mathrm{s}}} e_{i_{\mathrm{sx}}}+\omega_{\mathrm{g}} e_{i_{\mathrm{sy}}}+\frac{L_{\mathrm{m}} R_{\mathrm{r}}}{\sigma L_{\mathrm{s}} L_{\mathrm{r}}^{2}} e_{\psi_{\mathrm{rx}}}+\frac{L_{\mathrm{m}}}{\sigma L_{\mathrm{s}} L_{\mathrm{r}}} \omega_{\mathrm{r}} e_{\psi_{\mathrm{ry}}} \\
-\omega_{\mathrm{g}} e_{i_{\mathrm{sx}}}-\frac{R_{\mathrm{s}}^{\prime}}{\sigma L_{\mathrm{s}}} e_{i_{\mathrm{sy}}}-\frac{L_{\mathrm{m}}}{\sigma L_{\mathrm{s}} L_{\mathrm{r}}} \omega_{\mathrm{r}} e_{\psi_{\mathrm{rx}}}+\frac{L_{\mathrm{m}} R_{\mathrm{r}}}{\sigma L_{\mathrm{s}} L_{\mathrm{r}}^{2}} e_{\psi_{\mathrm{ry}}}
\end{array}\right]+\left[\begin{array}{cc}
\frac{1}{\sigma L_{\mathrm{s}}} & 0 \\
0 & \frac{1}{\sigma L_{\mathrm{s}}}
\end{array}\right]\left[\begin{array}{c}
e_{u_{\mathrm{sx}}} \\
e_{u_{\mathrm{sy}}}
\end{array}\right] \\
\dot{z}=\left[\begin{array}{l}
\dot{e}_{\psi_{\mathrm{rx}}} \\
\dot{e}_{\psi_{\mathrm{ry}}}
\end{array}\right]=\left[\begin{array}{l}
R_{\mathrm{r}} \frac{L_{\mathrm{m}}}{L_{\mathrm{r}}} e_{i_{\mathrm{sx}}}-\frac{R_{\mathrm{r}}}{L_{\mathrm{r}}} e_{\psi_{\mathrm{rx}}}+\left(\omega_{\mathrm{g}}-\omega_{\mathrm{r}}\right) e_{\psi_{\mathrm{ry}}} \\
R_{\mathrm{r}} \frac{L_{\mathrm{m}}}{L_{\mathrm{r}}} e_{i_{\mathrm{sy}}}-\left(\omega_{\mathrm{g}}-\omega_{\mathrm{r}}\right) e_{\psi_{\mathrm{rx}}}-\frac{R_{\mathrm{r}}}{L_{\mathrm{r}}} e_{\psi_{\mathrm{ry}}}
\end{array}\right]
\end{gathered}
$$

\section{CONTROLLERS DESIGN BY PASSIVE EQUIVALENCE}

\subsection{Concepts and basic definitions}

In this section, we recall some basic concepts on passive systems used in this study and given in Reference [28]. We will follow the same notation of [28] as far as possible.

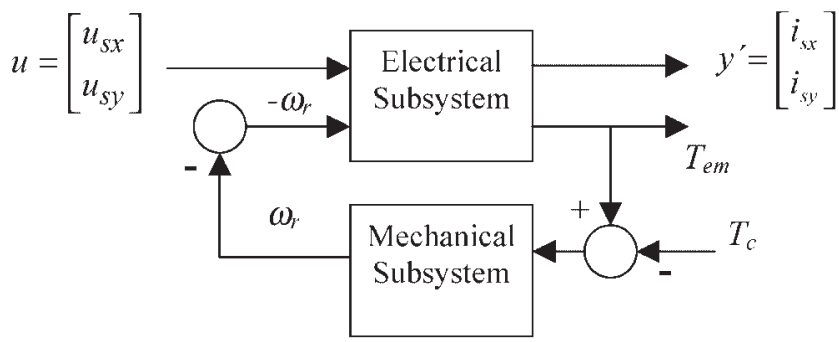

$u$ : system input.

$y^{\prime}$ : system output.

Figure 1. Electrical system to be controlled with a passive disturbance. 
Let us consider an $n$ th-order MIMO non-linear system of the form

$$
\begin{aligned}
& \dot{x}=f(x)+g(x) u \\
& y=h(x)
\end{aligned}
$$

with state space $X=\mathfrak{R}^{n}, u(t) \in U=\mathfrak{R}^{m}$ and $y(t) \in Y=\mathfrak{R}^{m}$. Moreover, $f \in \mathfrak{R}^{n}$ and the columns $g_{\mathrm{i}} \in \mathfrak{R}^{n}$ of $g \in \mathfrak{R}^{n \times m}$ are smooth vector fields (i.e. $C^{\infty}$ ) and $h \in \mathfrak{R}^{m}$ is a smooth mapping. It is assumed that vector field $f$ has at least one equilibrium point and without loss of generality will be assumed that $f(0)=0$ and $h(0)=0$.

\section{Definition 2.1}

System (4) is said to be $C^{r}$-passive if there exists a $C^{r}$ non-negative function $V: \Re^{n} \rightarrow \Re$, called storage function with $V(0)=0$, such that for all admissible $u \in U$, all initial condition $x(0)$ and all $t \geqslant 0$,

$$
V(x(t))-V(x(0)) \leqslant \int_{0}^{t} y^{\mathrm{T}}(s) u(s) \mathrm{d} s
$$

\section{Definition 2.2}

The system (4) is said to be locally feedback equivalent to a $C^{r}$-passive system (or just locally feedback $C^{r}$-passive) if there exists a feedback law of the form $u(t)=\alpha(x)+\beta(x) \varpi(t)$, where $\alpha(x)$ and $\beta(x)$ are smooth functions defined near $x=0$, with $\beta(x)$ invertible for all $x$, such that the system from the new input $\varpi(t)$ to the output $y(t)$ is $C^{r}$-passive. Equivalently

$$
\dot{V}(x)=\left(\frac{\partial V(x)}{\partial x}\right)^{\mathrm{T}}[f(x)+g(x) \alpha(x)+g(x) \beta(x) \varpi] \leq y^{\mathrm{T}} \varpi
$$

where $V$ is any $\mathrm{C}^{\mathrm{r}}$-storage function.

If we assume that $\operatorname{Lgh}(x)$ is non-singular in a neighbourhood of $x=0$, system (4) has a socalled relative degree $\rho=[1,1,1, \ldots .1]$ around $x=0$. Furthermore, if the distribution spanned by the vector fields $g_{1}(x), g_{2}(x), \ldots, g_{m}(x)$ is involutive, then there exists a new set of local co-ordinates $z(x) \in \mathfrak{R}^{n-m}$ defined around $x=0$ and vanishing in $x=0$, which together with the $m$ co-ordinates of the output $y(x)=h(x)$ allow to represent the system in its normal form [28] as follows:

$$
\begin{aligned}
& \dot{y}=a(y, z))+b(y, z) u \\
& \dot{z}=c(y, z)
\end{aligned}
$$

where $b(y, z)$ is invertible for all $(y, z)$ near $(0,0)$. We can express the system in terms of the socalled zero dynamics $f_{0}(z)$, defined as those internal dynamic which are consistent with the external constraint $y=0$ (in the case of system (4) the zero dynamics is defined by $\dot{z}=c \times$ $\left.(0, z)=f_{0}(z)\right)$. Thus, we can write (4) as

$$
\begin{aligned}
& \dot{y}=a(y, z))+b(y, z) u \\
& \dot{z}=f_{0}(z)+p(y, z) y
\end{aligned}
$$

where $p(y, z)$ is a smooth matrix of dimension $(n-m) \times m$.

If $\operatorname{Lgh}(0)$ is non-singular and the zero dynamics is such that the equilibrium point $z=0$ is asymptotically stable near $z=0$, system (4) is said to be locally minimum phase. 
Definition 2.3

A system (4) is said to be weakly locally minimum phase if there exists a positive definite $C^{r}$ function $W_{0}(z)$, with $r \geqslant 2$, locally defined near $z=0$ with $W_{0}(0)=0$, such that $L_{f_{0}} W_{0}(z) \leqslant 0$ for all $z$ in a neighbourhood of $z=0$.

A geometric characterization of systems that can be made $C^{2}$-passive is given in Reference [28]. They proved that system (4) can be made locally feedback equivalent to a $C^{2}$-passive system if and only if system (4) has unity relative degree $\rho=[1,1,1, \ldots .1]$ at $x=0$ and is locally weakly minimum phase.

\subsection{Model adjustments}

In order to apply the Theorems given in References [25,26], the electrical subsystem (3) must be parametrized in the normal form [28] with linear explicit parametric dependence of the form

$$
\begin{aligned}
& \dot{y}=\Lambda_{a} A(y, z)+\Lambda_{b} B(y, z) u \\
& \dot{z}=\Lambda_{0} f_{0}(z)+P^{\mathrm{T}}(y, z) \Lambda_{p} y
\end{aligned}
$$

with $z \in \mathfrak{R}^{2}, y \in \mathfrak{R}^{2}, u \in \mathfrak{R}^{2}, A(y, z) \in \mathfrak{R}^{8}, B(y, z) \in \mathfrak{R}^{2 \times 2}, f_{0} \in \mathfrak{R}^{2}, P(y, z) \in \mathfrak{R}^{2 \times 2}$. The parameters $\Lambda_{a} \in \mathfrak{R}^{2 \times 8}, \quad \Lambda_{b} \in \mathfrak{R}^{2 \times 2}, \quad \Lambda_{0} \in \mathfrak{R}^{2 \times 2}$ and $\Lambda_{p} \in \mathfrak{R}^{2 \times 2}$ represent constant but unknown parameters from a bounded compact set $\Omega$. Comparing Equations (3) and (5) we write the systems as

$$
\begin{aligned}
& \dot{y}=\left[\begin{array}{cccccccc}
-\frac{R_{\mathrm{s}}^{\prime}}{\sigma L_{\mathrm{s}}} & 0 & 0 & 1 & \frac{L_{\mathrm{m}} R_{\mathrm{r}}}{\sigma L_{\mathrm{s}} L_{\mathrm{r}}^{2}} & 0 & 0 & -\frac{L_{\mathrm{m}}}{\sigma L_{\mathrm{s}} L_{\mathrm{r}}} \\
0 & -\frac{R_{\mathrm{s}}^{\prime}}{\sigma L_{\mathrm{s}}} & -1 & 0 & 0 & \frac{L_{\mathrm{m}} R_{\mathrm{r}}}{\sigma L_{\mathrm{s}} L_{\mathrm{r}}^{2}} & \frac{L_{\mathrm{m}}}{\sigma L_{\mathrm{s}} L_{\mathrm{r}}} & 0
\end{array}\right]\left[\begin{array}{c}
e_{i_{\mathrm{sx}}} \\
e_{i_{\mathrm{sy}}} \\
\omega_{g} e_{i_{\mathrm{sx}}} \\
\omega_{g} e_{i_{\mathrm{sy}}} \\
e_{\psi_{\mathrm{rx}}} \\
e_{\psi_{\mathrm{ry}}} \\
\omega_{\mathrm{r}} e_{\psi_{\mathrm{rx}}} \\
\omega_{\mathrm{r}} e_{\psi_{\mathrm{ry}}}
\end{array}\right]+\left[\begin{array}{cc}
\frac{1}{\sigma L_{\mathrm{s}}} & 0 \\
0 & \frac{1}{\sigma L_{\mathrm{s}}}
\end{array}\right] I_{2} u \\
& \dot{z}=\left[\begin{array}{cc}
-\frac{R_{\mathrm{r}}}{L_{\mathrm{r}}} & \left(\omega_{\mathrm{g}}-\omega_{\mathrm{r}}\right) \\
-\left(\omega_{\mathrm{g}}-\omega_{\mathrm{r}}\right) & -\frac{R_{\mathrm{r}}}{L_{\mathrm{r}}}
\end{array}\right]\left[\begin{array}{l}
e_{\psi_{\mathrm{rx}}} \\
e_{\psi_{\mathrm{ry}}}
\end{array}\right]+I_{2}\left[\begin{array}{cc}
\frac{L_{\mathrm{m}}}{T_{\mathrm{r}}} & 0 \\
0 & \frac{L_{\mathrm{m}}}{T_{\mathrm{r}}}
\end{array}\right] y \\
& \text { with } z=\left[\begin{array}{c}
e_{\psi_{\mathrm{rx}}} \\
e_{\psi_{\mathrm{ry}}}
\end{array}\right], \quad y=\left[\begin{array}{c}
e_{i_{\mathrm{sx}}} \\
e_{i_{\mathrm{sy}}}
\end{array}\right], \quad u=\left[\begin{array}{c}
e_{u_{\mathrm{sx}}} \\
e_{u_{\mathrm{sy}}}
\end{array}\right]
\end{aligned}
$$


with $T_{\mathrm{r}}=L_{\mathrm{r}} / R_{\mathrm{r}}$. For this particular system representation, we can immediately identify the following terms:

$$
\begin{aligned}
& \Lambda_{a}=\left[\begin{array}{cccccccc}
-\frac{R_{\mathrm{s}}^{\prime}}{\sigma L_{\mathrm{s}}} & 0 & 0 & 1 & \frac{L_{\mathrm{m}} R_{\mathrm{r}}}{\sigma L_{\mathrm{s}} L_{\mathrm{r}}^{2}} & 0 & 0 & -\frac{L_{\mathrm{m}}}{\sigma L_{\mathrm{s}} L_{\mathrm{r}}} \\
0 & -\frac{R_{\mathrm{s}}^{\prime}}{\sigma L_{\mathrm{s}}} & -1 & 0 & 0 & \frac{L_{\mathrm{m}} R_{\mathrm{r}}}{\sigma L_{\mathrm{s}} L_{\mathrm{r}}^{2}} & \frac{L_{\mathrm{m}}}{\sigma L_{\mathrm{s}} L_{\mathrm{r}}} & 0
\end{array}\right], \quad A(y, z)=\left[\begin{array}{c}
e_{i_{\mathrm{sx}}} \\
e_{i_{\mathrm{sy}}} \\
\omega_{g} e_{i_{\mathrm{sx}}} \\
\omega_{g} e_{i_{\mathrm{sy}}} \\
e_{\Psi_{\mathrm{rx}}} \\
e_{\Psi_{\mathrm{ry}}} \\
\omega_{r} e_{\Psi_{\mathrm{rx}}} \\
\omega_{r} e_{\Psi_{\mathrm{ry}}}
\end{array}\right] \\
& \Lambda_{b}=\left[\begin{array}{cc}
\frac{1}{\sigma L_{\mathrm{s}}} & 0 \\
0 & \frac{1}{\sigma L_{\mathrm{s}}}
\end{array}\right], \quad B(y, z)=\left[\begin{array}{ll}
1 & 0 \\
0 & 1
\end{array}\right]=I_{2} \\
& \Lambda_{0}=\left[\begin{array}{cc}
-\frac{R_{\mathrm{r}}}{L_{\mathrm{r}}} & -\omega_{\mathrm{g}} \\
\omega_{\mathrm{g}} & -\frac{R_{\mathrm{r}}}{L_{\mathrm{r}}}
\end{array}\right], \quad f_{0}(z)=\left[\begin{array}{c}
e_{\Psi_{\mathrm{rx}}} \\
e_{\Psi_{\mathrm{ry}}}
\end{array}\right], \quad \Lambda_{\mathrm{p}}=\left[\begin{array}{cc}
\frac{L_{\mathrm{m}}}{T_{\mathrm{r}}} & 0 \\
0 & \frac{L_{\mathrm{m}}}{T_{\mathrm{r}}}
\end{array}\right], \quad P(y, z)=\left[\begin{array}{ll}
1 & 0 \\
0 & 1
\end{array}\right]=I_{2}
\end{aligned}
$$

Now it is necessary to check if subsystem (5) and (6) satisfy the three assumptions given in References $[25,26]$. First, we need to prove that the subsystem is locally weekly minimum phase by finding a positive definite differentiable function $W_{0}(z)$ satisfying $\left[\partial W_{0}(z) / \partial z\right]^{\mathrm{T}} \Lambda_{0} f_{0} \times$ $(z) \leqslant 0 \forall \Lambda_{0}$. If we choose the function: $W_{0}(z)=\frac{1}{2}\left(e_{\psi_{\mathrm{rx}}}^{2}+e_{\psi_{\mathrm{ry}}}^{2}\right)=\frac{1}{2} z^{\mathrm{T}} z$, then $\partial W_{0}(z) / \partial z=$ $\left[\begin{array}{ll}e_{\psi_{\mathrm{rx}}} & e_{\psi_{\mathrm{ry}}}\end{array}\right]^{\mathrm{T}}=z^{\mathrm{T}}$.

It can be checked that:

$$
\begin{aligned}
{\left[\frac{\partial W_{0}(z)}{\partial z}\right]^{\mathrm{T}} \Lambda_{0} f_{0}(z) } & =\left[\begin{array}{ll}
e_{\psi_{\mathrm{rx}}} & e_{\psi_{\mathrm{ry}}}
\end{array}\right]\left[\begin{array}{c}
-\frac{R_{\mathrm{r}}}{L_{\mathrm{r}}} e_{\psi_{\mathrm{rx}}}+\omega_{\mathrm{g}} e_{\psi_{\mathrm{ry}}} \\
-\omega_{\mathrm{g}} e_{\psi_{\mathrm{rx}}}-\frac{R_{\mathrm{r}}}{L_{\mathrm{r}}} e_{\psi_{\mathrm{ry}}}
\end{array}\right] \\
& =-\frac{R_{\mathrm{r}}}{L_{\mathrm{r}}} e_{\psi_{\mathrm{rx}}}^{2}+\omega_{\mathrm{g}} e_{\psi_{\mathrm{rx}}} e_{\psi_{\mathrm{ry}}}-\omega_{\mathrm{g}} e_{\psi_{\mathrm{rx}}} e_{\psi_{\mathrm{ry}}}-\frac{R_{\mathrm{r}}}{L_{\mathrm{r}}} e_{\psi_{\mathrm{ry}}}^{2} \\
& =-\frac{R_{\mathrm{r}}}{L_{\mathrm{r}}}\left(e_{\psi_{\mathrm{rx}}}^{2}+e_{\psi_{\mathrm{ry}}}^{2}\right) \leqslant 0
\end{aligned}
$$

concluding that subsystem (3) is locally weakly minimum phase. The second assumption from [25] is that matrix $B(y, z)$ should be invertible. From Equations (6) it can be readily checked that matrix $B(y, z)=I_{2 \times 2}$, is in fact invertible. The third assumption of the method has to do with the knowledge of the sing of matrix $\Lambda_{b}$. In this particular case from Equations (6) it can be seen that since matrix $\Lambda_{b}$ is diagonal we have $\Lambda_{b}=\operatorname{sign}\left(\Lambda_{b}\right)\left|\Lambda_{b}\right|$ with $\operatorname{sign}\left(\Lambda_{b}\right)=I_{2 \times 2}$. 


\subsection{Proposed controller with fixed adaptive gains ( $M I M O_{-} C F A G$ )}

According to [25] there exist and adaptive controller denoted as MIMO_CFAG of the form

$$
u(t)=\left[\theta_{1}(t) A(y, z)-\theta_{2}(t) P(y, z) \frac{\partial W_{0}(z)}{\partial z}+\theta_{3}(t) \varpi(t)\right]
$$

with the adaptive laws:

$$
\begin{aligned}
& \dot{\theta}_{1}(t)=-y A^{\mathrm{T}}(y, z) \\
& \dot{\theta}_{2}(t)=-y\left(\frac{\partial W_{0}(z)}{\partial z}\right)^{\mathrm{T}} P^{\mathrm{T}}(y, z) \\
& \dot{\theta}_{3}(t)=-y \omega^{\mathrm{T}}(t)
\end{aligned}
$$

that applied to subsystem (3) makes the system locally feedback equivalent to a $C^{2}$-passive subsystem from the input $\varpi(t)$ to the output $y(t)$. The parameters $\theta_{1}(t) \in \mathfrak{R}^{2 \times 8}, \theta_{2}(t) \in \mathfrak{R}^{2 \times 2}$ and $\theta_{3}(t) \in \mathfrak{R}^{2 \times 2}$ represent adjustable controller parameters whose ideal values are $\theta_{1}^{*}=-\Lambda_{b}^{-1} \Lambda_{a} \in \mathfrak{R}^{2 \times 8}, \theta_{2}^{*}=-\Lambda_{b}^{-1} \Lambda_{p}^{\mathrm{T}} \in \mathfrak{R}^{2 \times 2}$ and $\theta_{3}^{*}=-\Lambda_{b}^{-1} \in \mathfrak{R}^{2 \times 2}$.

\subsection{Proposed controller with time-varying adaptive gains (MIMO_CTVAG)}

Another adaptive controller with time varying gains can also be proposed for this case [26]. This controller, denoted as MIMO_CTVAG, has the following form:

$$
u(t)=\left[\theta_{1}(t) A(y, z)+\theta_{2}(t) P(y, z) \frac{\partial W_{0}(z)}{\partial z}+\theta_{3}(t) \varpi(t)\right]
$$

with adaptive laws given by:

$$
\begin{gathered}
\dot{\theta}_{1}(t)=-y A^{\mathrm{T}}(y, z) \frac{\Gamma_{1}^{-1}}{\sqrt{1+\operatorname{Trace}\left(\Gamma_{1}^{-2}+\Gamma_{2}^{-2}+\Gamma_{3}^{-2}\right)}} \\
\dot{\theta}_{2}(t)=-\frac{\Gamma_{2}^{-1}}{\sqrt{1+\operatorname{Trace}\left(\Gamma_{1}^{-2}+\Gamma_{2}^{-2}+\Gamma_{3}^{-2}\right)}} y\left(\frac{\partial W_{0}(z)}{\partial z_{1}}\right)^{\mathrm{T}} P^{\mathrm{T}}(y, z) \\
\dot{\theta}_{3}(t)=-\frac{\Gamma_{3}^{-1}}{\sqrt{1+\operatorname{Trace}\left(\Gamma_{1}^{-2}+\Gamma_{2}^{-2}+\Gamma_{3}^{-2}\right)}} y \varpi^{\mathrm{T}}(t)
\end{gathered}
$$

and time-varying adaptive gains defined by:

$$
\begin{array}{ll}
\dot{\Gamma}_{1}=-\Gamma_{1} A(y, z) A^{\mathrm{T}}(y, z) \Gamma_{1}, & \Gamma_{1}\left(t_{0}\right)>0 \\
\dot{\Gamma}_{2}=-\Gamma_{2} P(y, z)\left(\frac{\partial W_{0}(z)}{\partial z}\right)\left(\frac{\partial W_{0}(z)}{\partial z}\right)^{\mathrm{T}} P^{\mathrm{T}}(y, z) \Gamma_{2}, & \Gamma_{2}\left(t_{0}\right)>0 \\
\dot{\Gamma}_{3}=-\Gamma_{3} \varpi(t) \varpi^{\mathrm{T}}(t) \Gamma_{3}, & \Gamma_{3}\left(t_{0}\right)>0
\end{array}
$$

According to [26], this controller applied to subsystem (3) will convert it in an equivalent $C^{2}$ passive system from the input $\varpi(t)$ to the output $y(t)$. The parameters $\theta_{1}(t) \in \mathfrak{R}^{2 \times 8}, \theta_{2}(t) \in \mathfrak{R}^{2 \times 2}$ and $\theta_{3}(t) \in \mathfrak{R}^{2 \times 2}$ represent adjustable controller parameters whose ideal values are $\theta_{1}^{*}=$ $-\Lambda_{b}^{-1} \Lambda_{a} \in \mathfrak{R}^{2 \times 8}, \theta_{2}^{*}=-\Lambda_{b}^{-1} \Lambda_{p}^{\mathrm{T}} \in \mathfrak{R}^{2 \times 2}$ and $\theta_{3}^{*}=-\Lambda_{b}^{-1} \in \mathfrak{R}^{2 \times 2}$. 
By applying the MIMO_CFAG given by Equations (7) and (8) and MIMO_CTVAG given by Equations (9)-(11), we will obtain two control schemes that do not need the knowledge of any motor-load parameters. Because the control laws and the adaptive laws depend on $\partial W_{0} \times$ $(z) / \partial z=z$, in these control schemes it is necessary to know the error of rotor flux components, i.e. rotor flux component estimations or measurements. However, based on the principle stated in Section 4, rotor flux estimation will be not necessary, providing a considerable simplification in the controllers.

\section{PRINCIPLE OF TORQUE-FLUX CONTROL}

In Reference [5] it is proposed the following principle which considerably simplifies the controller design and avoid the rotor current or rotor flux estimations.

\subsection{Principle statement}

\subsubsection{Principle of torque-flux control}

In the controller design of alternating current motors based on a model of the generalized electrical machine, working in a control scheme with the co-ordinate transformation block $\mathrm{e}^{\mathrm{j} \rho_{\mathrm{g}}}$ (Field Oriented Scheme) to transform from stationary to rotating co-ordinate system, in order to control the torque and flux the controller can be limited only to control the stator currents. So, it is useless to make efforts to directly control rotor flux or rotor current components. Note that the controller still guarantee a suitable control of the torque and flux and it is then possible to discard all the terms concerning the rotor current or rotor flux components in its design.

This principle is based on the fact that if the field orientation is being carried out, then the torque control is being done by the quadrature current controller and the flux control is being done by the direct current controller. Besides, the current controller will act in such a manner that $e_{i s \mathrm{y}} \rightarrow 0$ and $e_{i \mathrm{sx}} \rightarrow 0$. It should be noted that if $e_{i s \mathrm{y}} \rightarrow 0$ and $e_{i \mathrm{sx}} \rightarrow 0$, the last two equations of (3) tend to

$$
\dot{z}=\left[\begin{array}{c}
\dot{e}_{\psi_{\mathrm{rx}}} \\
\dot{e}_{\psi_{\mathrm{ry}}}
\end{array}\right]=\left[\begin{array}{c}
-\frac{R_{\mathrm{r}}}{L_{\mathrm{r}}} e_{\psi_{\mathrm{rx}}}+\left(\omega_{\mathrm{g}}-\omega_{\mathrm{r}}\right) e_{\psi_{\mathrm{ry}}} \\
-\left(\omega_{\mathrm{g}}-\omega_{\mathrm{r}}\right) e_{\psi_{\mathrm{rx}}}-\frac{R_{r}}{L_{r}} e_{\psi_{\mathrm{ry}}}
\end{array}\right]
$$

and can be written as

$$
\dot{z}=\left[\begin{array}{cc}
-\frac{R_{\mathrm{r}}}{L_{\mathrm{r}}} & \left(\omega_{\mathrm{g}}-\omega_{\mathrm{r}}\right) \\
-\left(\omega_{\mathrm{g}}-\omega_{\mathrm{r}}\right) & -\frac{R_{\mathrm{r}}}{L_{\mathrm{r}}}
\end{array}\right] z
$$

This last equation is a first-order non-linear dynamical system of the form $\dot{z}=A z$, which is asymptotically stable. In fact, let us choose the Lyapunov function candidate $V=\frac{1}{2} z^{\mathrm{T}} z$, whose 
time derivative is $\dot{V}=z^{\mathrm{T}} \dot{z} \leqslant 0$. Evaluating its time derivative along the system trajectory we get

$$
\dot{V}=-\frac{R_{\mathrm{r}}}{L_{\mathrm{r}}} z^{\mathrm{T}} z<0
$$

Since the system has a Lyapunov function $V$ positive definite with a time derivative $\dot{V}$ negative definite, then the system is asymptotically stable, provided $y=\left(e_{i \mathrm{sx}}, e_{i s \mathrm{y}}\right) \rightarrow 0$. This means that the flux components $z=\left(e_{\psi_{\mathrm{rx}}}, e_{\psi_{\mathrm{ry}}}\right)$ tend to zero asymptotically. In other words, the current controllers not only guarantee that $\left(e_{i \mathrm{sx}}, e_{i \mathrm{sy}}\right) \rightarrow 0$ when $t \rightarrow \infty$, but also guarantee that $z=\left(e_{\psi \mathrm{sx}}\right.$, $\left.e_{\psi \text { sy }}\right) \rightarrow 0$ when $t \rightarrow \infty$, provided the current controllers work properly. Therefore, the contributions of the terms involving the flux can be discarded in the controller design (i.e. $z=\left[e_{\psi_{\mathrm{rx}}} e_{\psi_{\mathrm{ry}}}\right]^{\mathrm{T}}=0$ in the control and adaptive laws).

\subsection{Application to controller MIMO_CFAG}

Applying the above principle to MIMO_CFAG, as already mention in Section 4.1, we can disregard the rotor flux components in the control law (7) (i.e. to consider $\left(\partial W_{o}(z) / \partial z\right)=z=0$ ). In this case it can be proved [25] that the storage function still assures that the resulting system is equivalent to a $C^{2}$-passive system. As a consequence, there is no need for adaptation of $\theta_{2}$ parameter in the controller. Thus the controller is simplified to

$$
\begin{aligned}
u(t) & =\theta_{1}(t)\left[\begin{array}{ll}
y^{\mathrm{T}} & \omega_{g} y^{\mathrm{T}}
\end{array}\right]+\theta_{3}(t) \varpi(t) \\
\dot{\theta}_{1}(t) & =-y\left[\begin{array}{ll}
y^{\mathrm{T}} & \omega_{g} y^{\mathrm{T}}
\end{array}\right] \\
\dot{\theta}_{3}(t) & =-y \varpi^{\mathrm{T}}(t)
\end{aligned}
$$

where $\theta_{1}(t) \in \mathfrak{R}^{2 \times 4}$ and $\theta_{3}(t) \in \mathfrak{R}^{2 \times 2}$ are the controller adjustable parameters whose ideal values are $\theta_{1}^{*}=-\Lambda_{b}^{-1} \Lambda_{a} \in \mathfrak{R}^{2 \times 2}$ and $\theta_{3}^{*}=-\Lambda_{b}^{-1} \in \mathfrak{R}^{2 \times 2}$.

On the other hand, a still more simplified controller is obtained by setting the controller directly in the stator co-ordinate system. This means that $\omega_{\mathrm{g}}=0$, so the following MIMO controller applicable to subsystem (3) is finally obtained:

$$
\begin{gathered}
u(t)=\theta_{1}(t) y+\theta_{3}(t) \varpi(t) \\
\dot{\theta}_{1}(t)=-y y^{\mathrm{T}} \\
\dot{\theta}_{3}(t)=-y \varpi^{\mathrm{T}}(t)
\end{gathered}
$$

where $\theta_{1}(t) \in \mathfrak{R}^{2 \times 2}$ and $\theta_{3}(t) \in \mathfrak{R}^{2 \times 2}$ are the controller adjustable parameters whose ideal values are $\theta_{1}^{*}=-\Lambda_{b}^{-1} \Lambda_{a} \in \mathfrak{R}^{2 \times 2}$ and $\theta_{3}^{*}=-\Lambda_{b}^{-1} \in \mathfrak{R}^{2 \times 2}$.

\subsection{Application to controller MIMO_CTVAG}

In the same way as in Section 4.2 it can be verified that applying the Torque-Flux Control Principle, the MIMO_CTVAG can be simplified to

$$
\begin{aligned}
u(t) & =\theta_{1}(t) y+\theta_{3}(t) \varpi(t) \\
\dot{\theta}_{1}(t) & =-\frac{\Gamma_{1}^{-1}}{\sqrt{1+\operatorname{Trace}\left(\Gamma_{1}^{-2}+\Gamma_{3}^{-2}\right)}} y y^{\mathrm{T}}
\end{aligned}
$$




$$
\begin{aligned}
\dot{\theta}_{3}(t) & =-\frac{\Gamma_{3}^{-1}}{\sqrt{1+\operatorname{Trace}\left(\Gamma_{1}^{-2}+\Gamma_{3}^{-2}\right)}} y \varpi^{\mathrm{T}}(t) \\
\dot{\Gamma}_{1} & =-\Gamma_{1} y y^{\mathrm{T}} \Gamma_{1}, \quad \Gamma_{1}\left(t_{0}\right)>0 \\
\dot{\Gamma}_{3} & =-\Gamma_{3} \varpi(t) \varpi^{\mathrm{T}}(t) \Gamma_{3}, \quad \Gamma_{3}\left(t_{0}\right)>0
\end{aligned}
$$

where $\theta_{1}(t) \in \mathfrak{R}^{2 \times 2}$ and $\theta_{3}(t) \in \mathfrak{R}^{2 \times 2}$ are the controller adjustable parameters whose ideal values are $\theta_{1}^{*}=-\Lambda_{b}^{-1} \Lambda_{a} \in \mathfrak{R}^{2 \times 2}$ and $\theta_{3}^{*}=-\Lambda_{b}^{-1} \in \mathfrak{R}^{2 \times 2}$.

\section{SIMULATION RESULTS}

In order to verify the advantages of the proposed controllers, the results obtained from the proposed controllers here and those presented in Reference [5] are compared. For the sake of space only the MIMO results are shown. The reader is referred to [5] for the case where two passive SISO adaptive controllers are used an compared with a classical control scheme given in Reference [27].

In simulations it was considered a squirrel-cage induction motor whose nominal parameters are: $15 \mathrm{~kW}(20 \mathrm{HP}), 220 \mathrm{~V}, \mathrm{fp}=0.853,4$ poles, $60 \mathrm{~Hz}, R_{\mathrm{s}}=0.1062 \Omega, X_{\mathrm{ls}}=X_{\mathrm{lr}}=0.2145 \Omega$, $x_{\mathrm{m}}=5.8339 \Omega, R_{\mathrm{r}}=0.0764 \Omega, \mathrm{J}=2.8 \mathrm{~kg} \mathrm{~m}^{2}, B_{\mathrm{p}}=0$. This induction motor was taken from [27]. All the simulations were made using the software package SIMULINK, MATLAB with ODE $15 \mathrm{~s}$ (stiff/NDF) integration method and variable step size.

The proposed control scheme is shown in Figure 2(a) as a representation for Matlab/Simulink block diagram. The two proposed controllers MIMO_CFAG and MIMO_CTVAG were developed and tested in the 'Proposed Controller' block of the scheme shown in Figure 2(b). It is important to observe that the speed, rotor flux, and stator current loop controllers are simple proportional (P) gains. These proportional gains were tuned such that the current, flux, speed and voltage were in suitable ranges. The control scheme obtained is similar to those proposed in Reference [5], the difference is in the controller employed in the 'Proposed Controller' block, being two SISO controllers in Reference [5] and being a MIMO controller in the scheme proposed here. This control scheme just need the exact values (or the estimates) of parameters $\mathrm{X}_{\mathrm{m}}$ and $\mathrm{T}_{\mathrm{r}}$ for the 'Field_orient' block. No other parameter or state estimations or measurements are needed.

In Figure 2(b), a general block diagram of the proposed control scheme is presented.

Figure 3 shows the information used to compare the control schemes. It is shown the variations of the reference speed $\omega_{\mathrm{r}}^{*}$, the variations in load torque, the variation of about $30 \%$ in the stator and rotor resistance, the linear increasing up to the double of the load inertia during the motor operation and the variations in the viscous friction coefficient. For both proposed controllers, five comparative tests considering the variations shown in Figure 3 were carried out. These tests will allow us to study the behaviour of the schemes under the following situations:

\section{Situations:}

(1) Initially, all the schemes are considered with the nominal motor data and with a reference speed increasing in a ramp fashion until $0.5 \mathrm{~s}$ and after that time remaining constant. Meanwhile, the load torque is kept constant. Form this initial Situation, several variations are then introduced. 

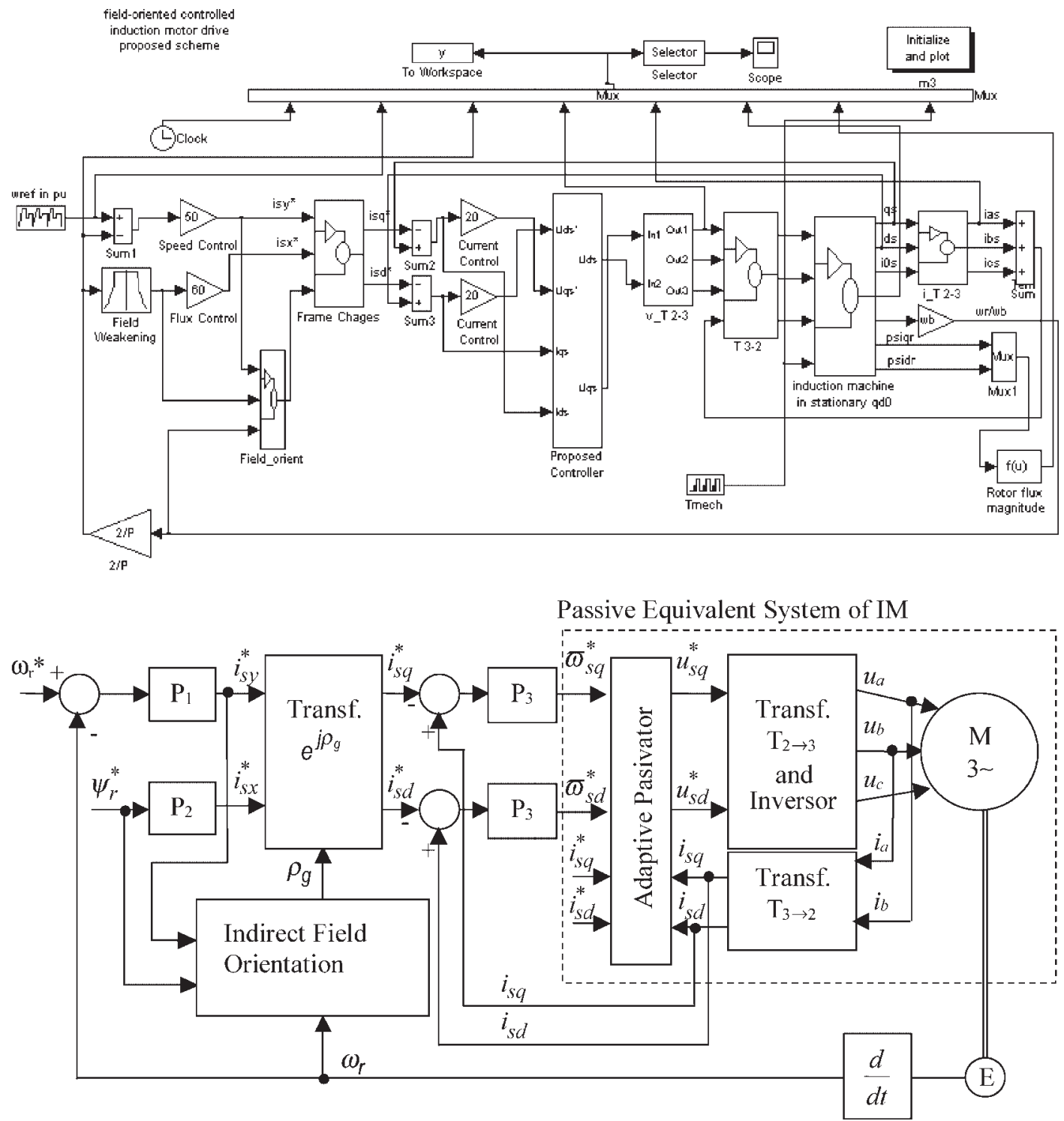

Figure 2. (a). Matalab/simulink block diagram of the proposed control scheme. (b). Block diagram of the proposed control scheme with field oriented block.

(2) Variations on load torque, as indicated in the Figure 3(b), are introduced.

(3) Variations on speed reference, as shown in the Figure 3(a), were studied.

(4) Variation of the motor resistances, as shown in Figures 3(c) and 3(d), are done.

(5) Variation of the load parameters, as indicated in Figures 3(e) and 3(f), were performed.

(6) Changes in the controller parameters (P) of control loops are analysed. 


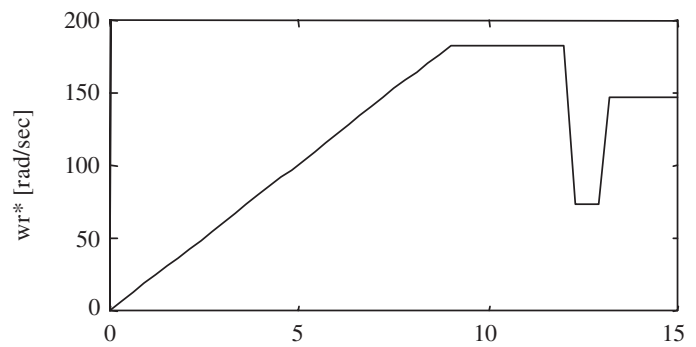

(a)
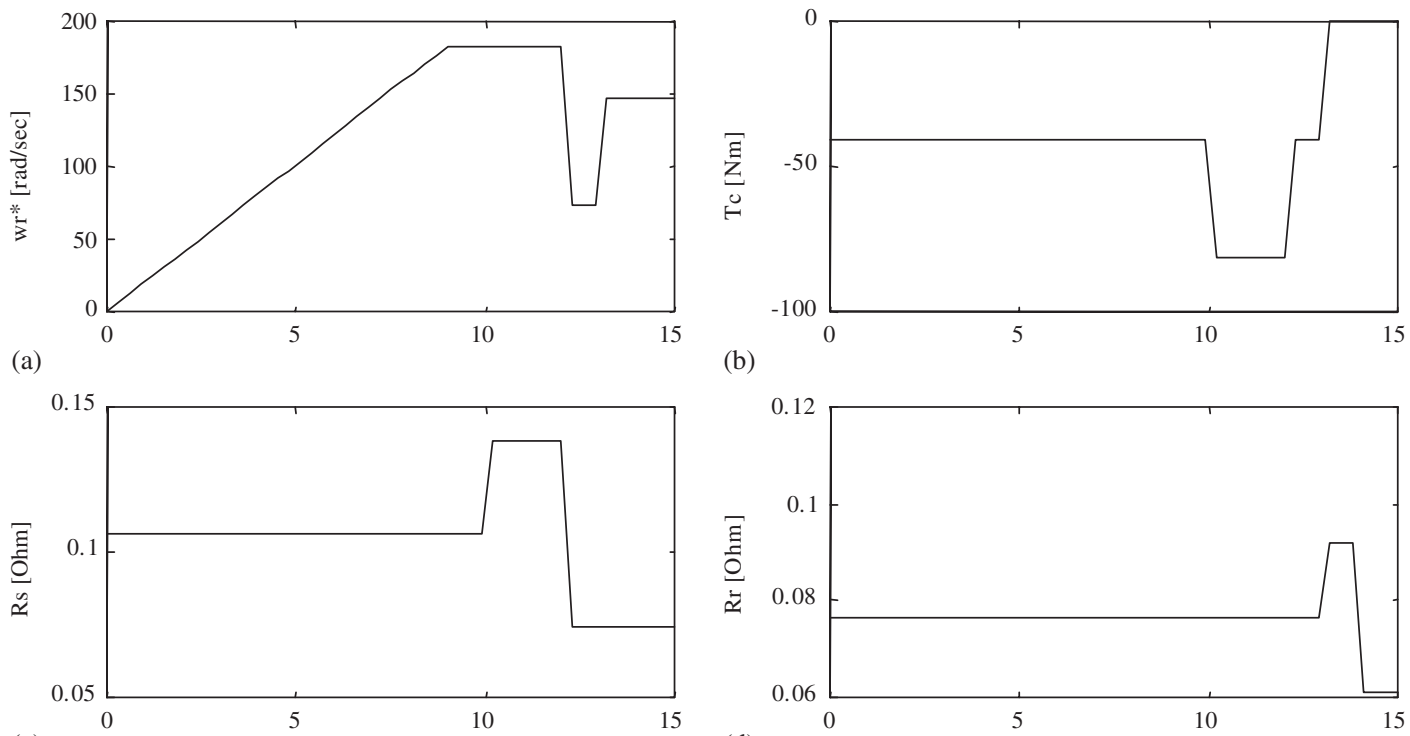

(b)

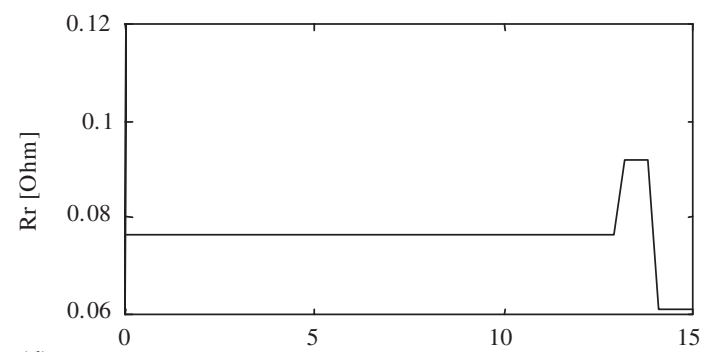

(d)
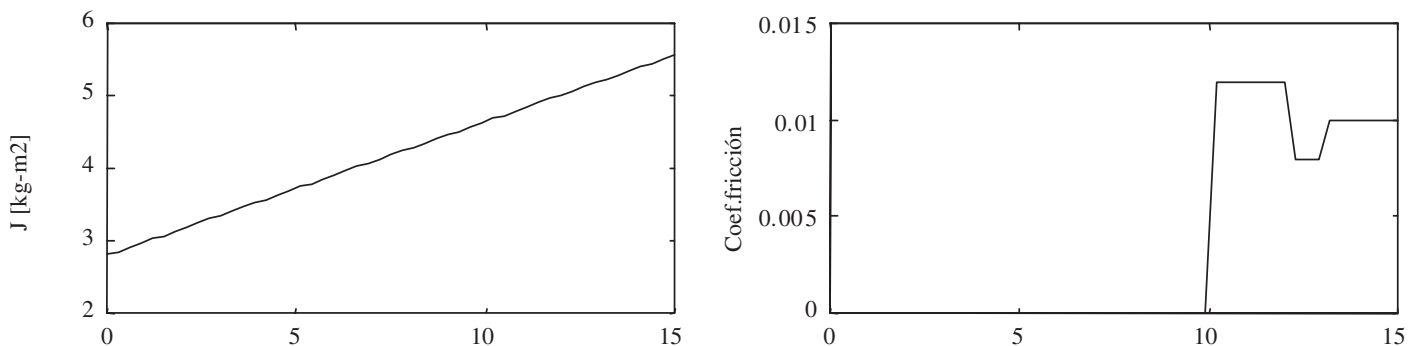

(f)

Figure 3. Parameter and reference variations used in the set of comparative tests.

In the simulation results of the proposed controllers shown in what follows, the initial conditions of all controller parameters were set to zero $\left(\theta_{i}(0)=0\right.$ for $\left.i=1,3\right)$ and the initial conditions of the adaptive gains were set equal to unity $\left(\Gamma_{i}(0)=I\right.$, for $\left.i=1,3\right)$.

Figure 4 shows the results obtained for the two proposed controllers under normal conditions (i.e. according to Situation 1), without considering variations of any type. From Figure 4, the controller MIMO_CFAG presents a quite accurate stationary state (with a velocity error lesser than $0.5 \%$ ). We can also see that the MIMO_CTVAG is equally accurate as the MIMO_CFAG, but a little less oscillatory.

Let us observe next in Figure 5, how the different schemes behave under variations of the load torque, as described in Figure 3(b). In the case of the MIMO_CFAG shown in Figure 5, the error values are $0.5 \%$ for a nominal load torque and of $0.22 \%$ for a half nominal load torque. The controller MIMO_CTVAG presents a similar response to that of MIMO_CFAG, but the transient response is slightly better. We also found that both controllers are less affected under abrupt variations of load torque than the classical scheme shown in Reference [5]. 


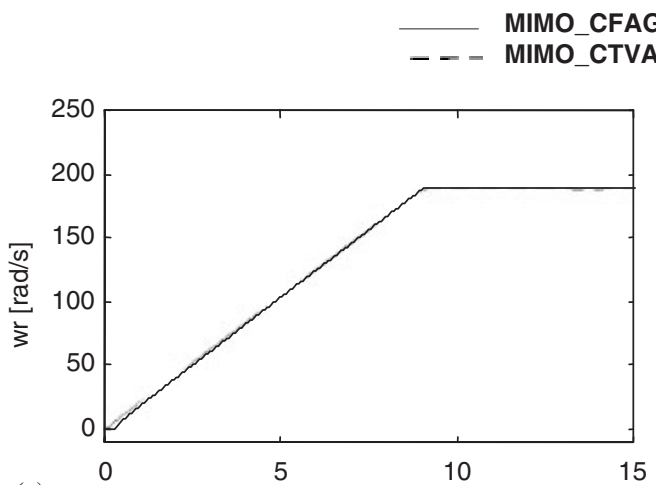

(a)

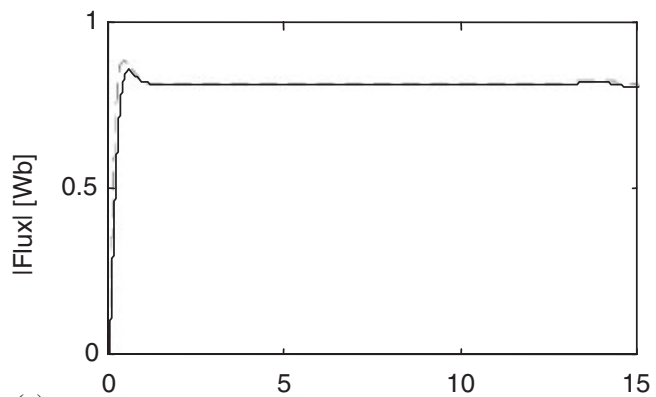

(c)

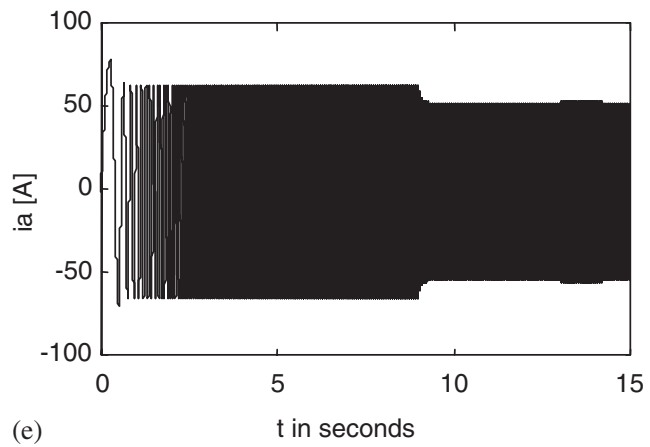

(b)
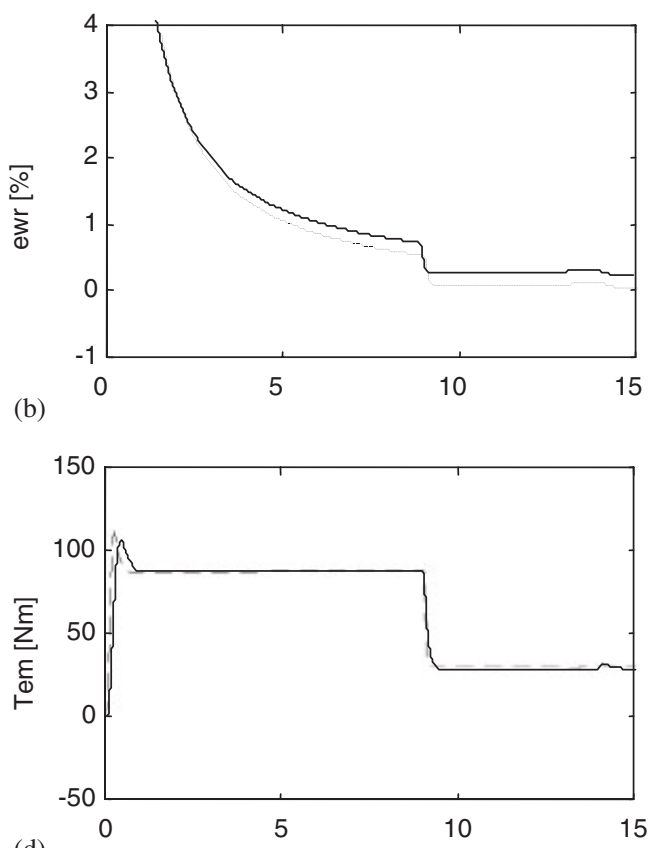

(d)

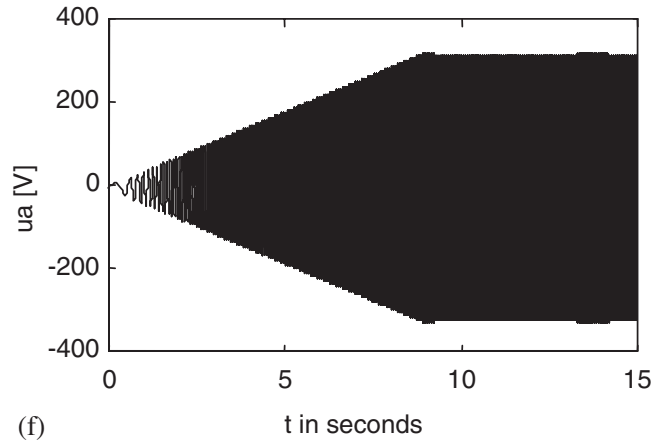

Figure 4. Results using MIMO_CFAG and MIMO_CTVAG for the initial situation.

In Figure 6, the effects of speed reference variations at nominal load torque, according to the variations indicated in Figure 3(a), are studied. The results for proposed controllers MIMO_CFAG and MIMO_CTVAG are similar to those obtained in the SISO case [5], rendering similar velocity errors whereas the rest of the variables present a suitable behaviour. In these cases, we have an error of about $0.5 \%$ for nominal speed and of approximately $0.8 \%$ at half of the nominal speed.

When analysing the Situation 4 (Figures 3(c) and 3(d)) both controllers present a good behaviour under changes on the stator resistance (See Figure 7). Nevertheless, under changes of the rotor resistance the right field orientation is lost and the speed response is considerably 


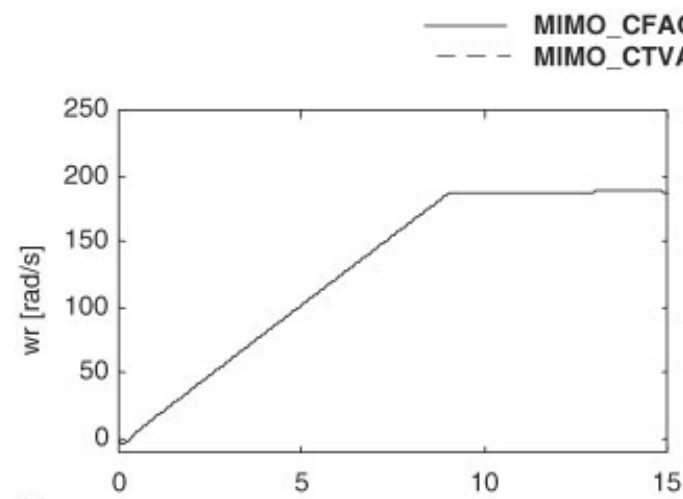

(a)

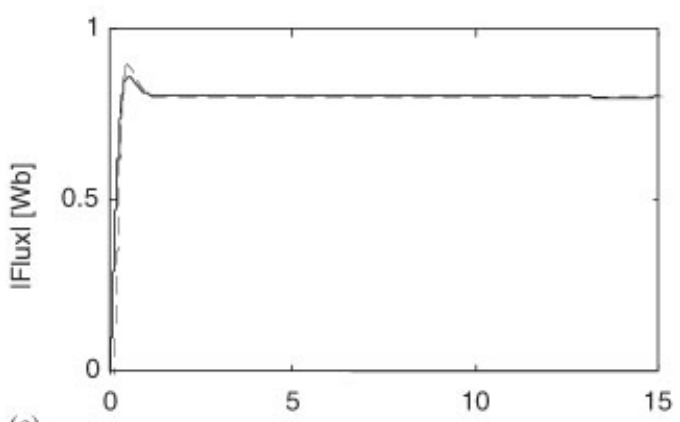

(c)

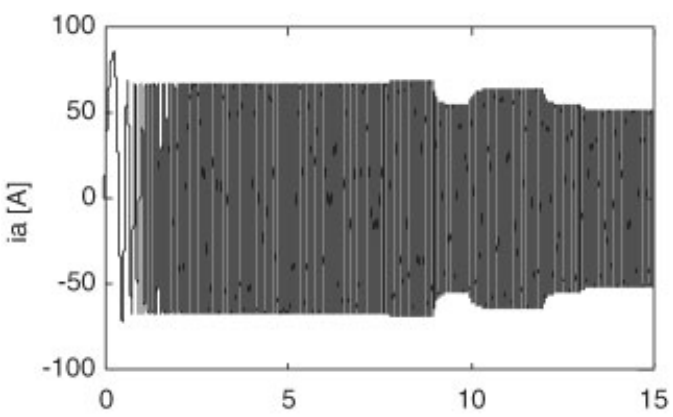

(e)

$\mathrm{t}$ in seconds

(b)
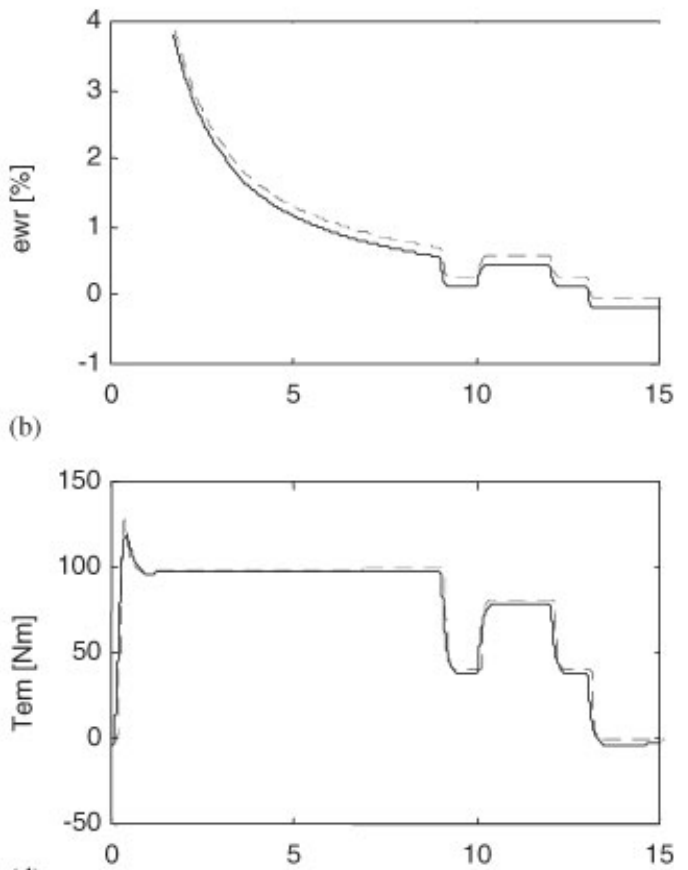

(d)

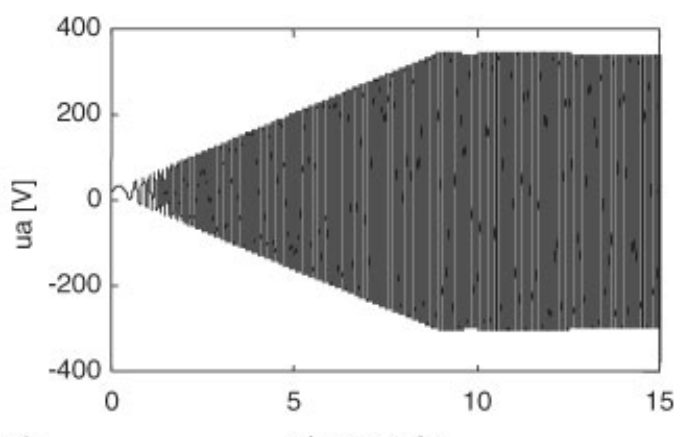

(f)

$\mathrm{t}$ in seconds

Figure 5. Results MIMO_CFAG and MIMO_CTVAG under variations of the load torque.

affected. Note how the flow of the machine diminishes considerably when the rotor resistance is decreased. We can also claim that the response in both cases are much more robust than the traditional controller shown in Reference [5]. Both controllers present lesser speed errors in steady state than the classical scheme studied in Reference [5].

Considering now the variations of the load parameters according to Situation 5 (Figures 3(e) and 3(f)), none of the two controllers under study were affected, as shown in Figure 8. For the proposed controllers, differences found in the general behaviour still remains. MIMO_CFAG presents a similar error in steady state than the SISO controllers studied in Reference [5]. 


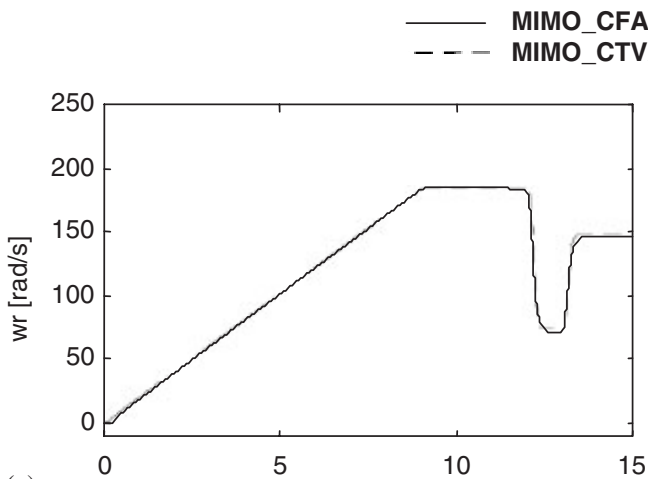

(a)
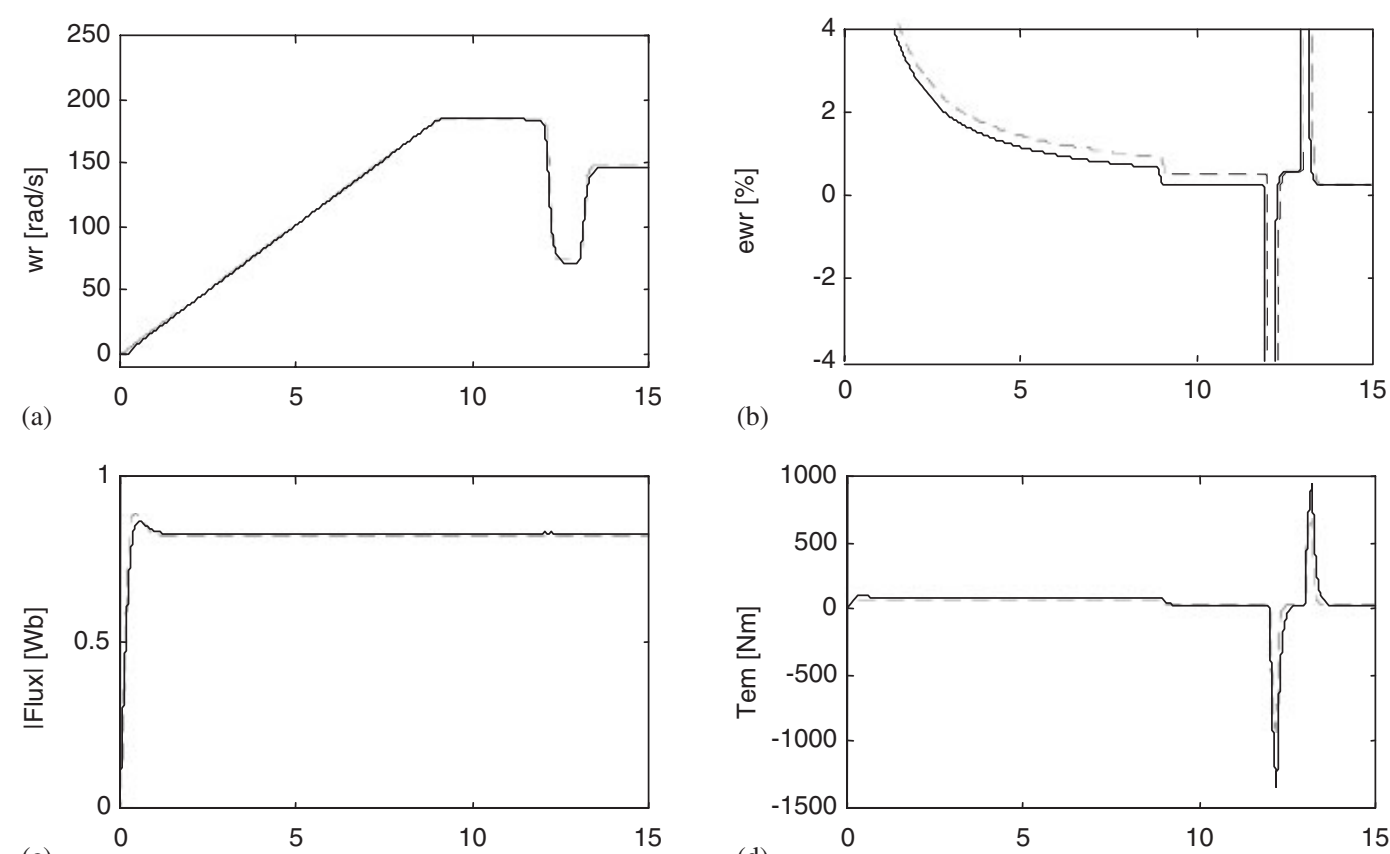

(b)

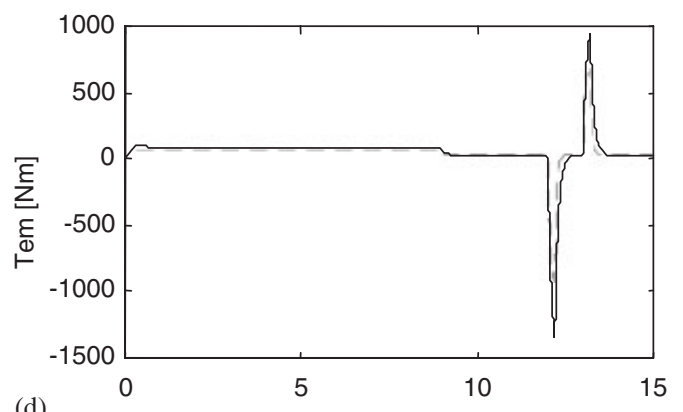

(d)

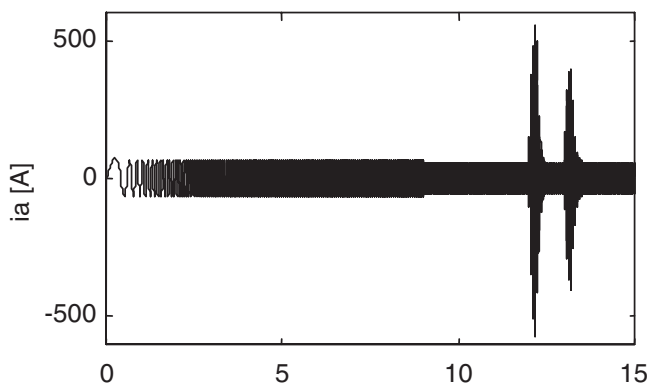

(e)

$t$ in seconds

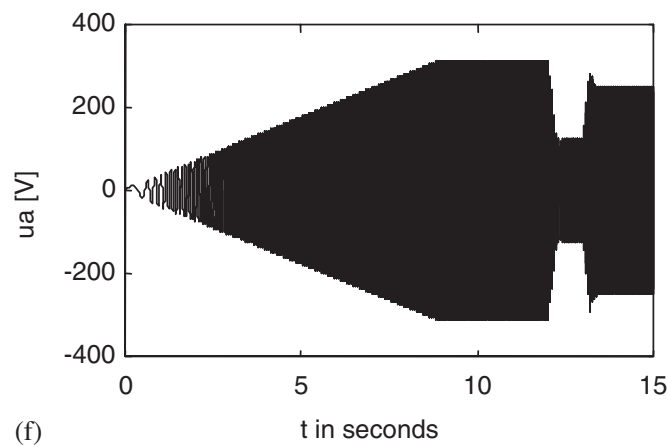

Figure 6. Results using MIMO_CFAG and MIMO_CTVAG under variations in the speed reference.

MIMO_CTVAG exhibits a response similar to that of the MIMO_CFAG but with a slightly better transient behaviour.

In Figure 9, the proportional gains of all control loops were changed. For both controllers MIMO_CFAG and MIMO_CTVAG, variations for the speed loop control parameter of $37.5 \%$ were applied ( $P$ varies from 80 to 50$)$. The flux loop was varied in 13\%, $(P$ changes from 69 to 60). The current loops were varied in 33.3\% ( $P$ varies from 30 to 20). From Figure 9 it can be seen how in spite of these simultaneous gain variations, the speed error continues being lesser than $1 \%$ and the transient response after $0.5 \mathrm{~s}$. was practically not affected. MIMO_CFAG as 


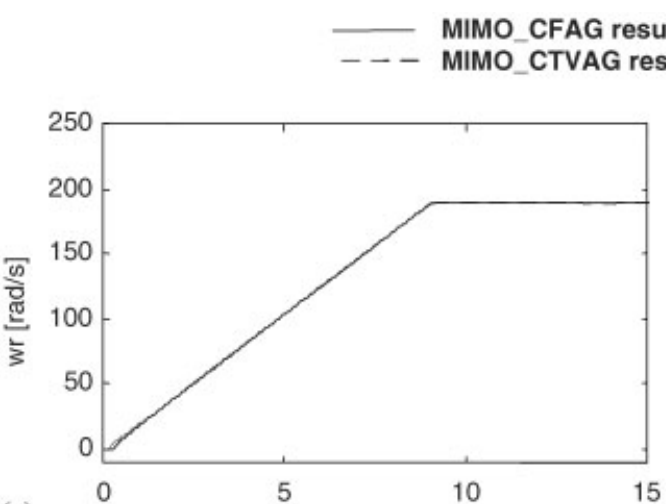

(a)
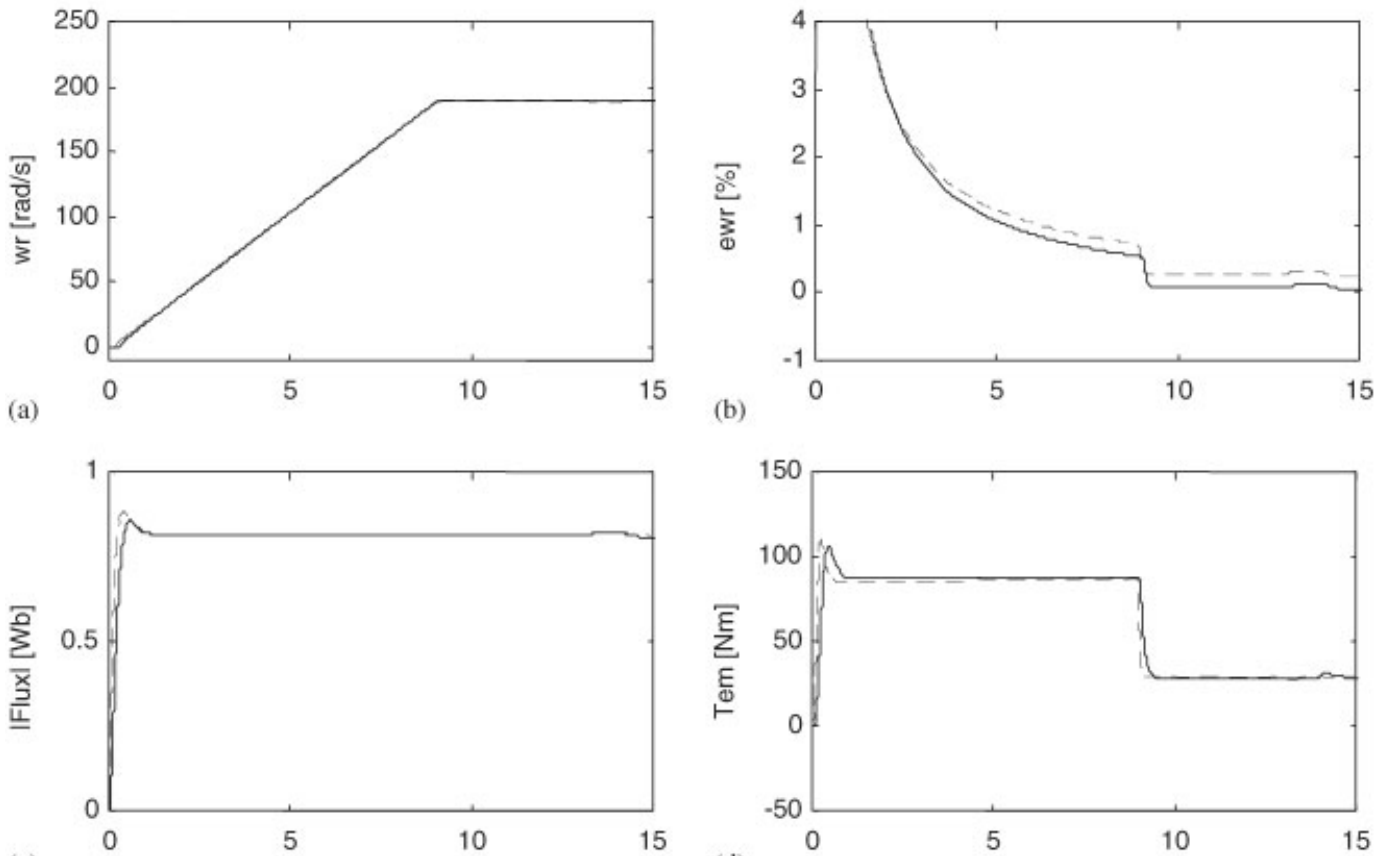

(c)
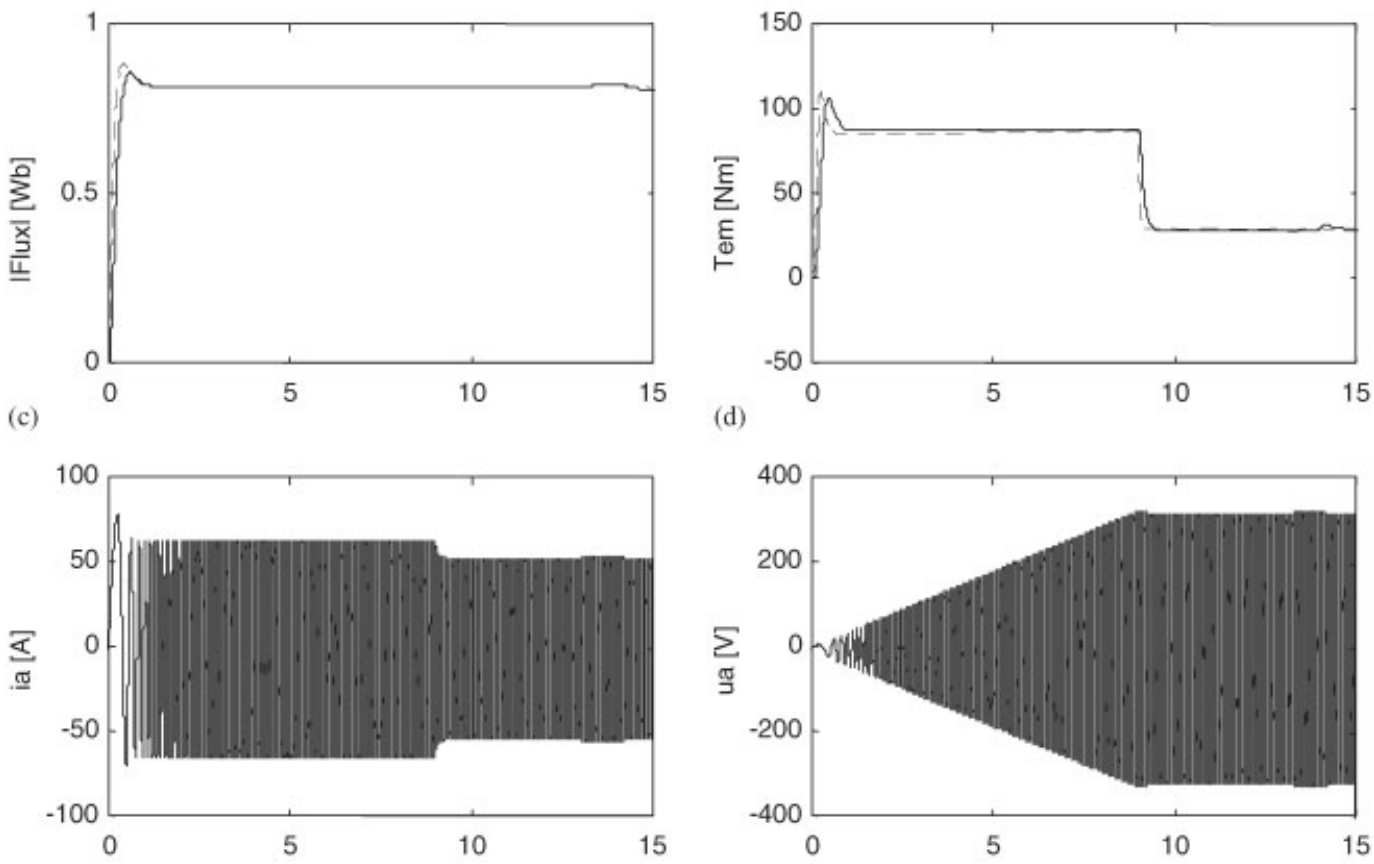

(e)

$t$ in seconds

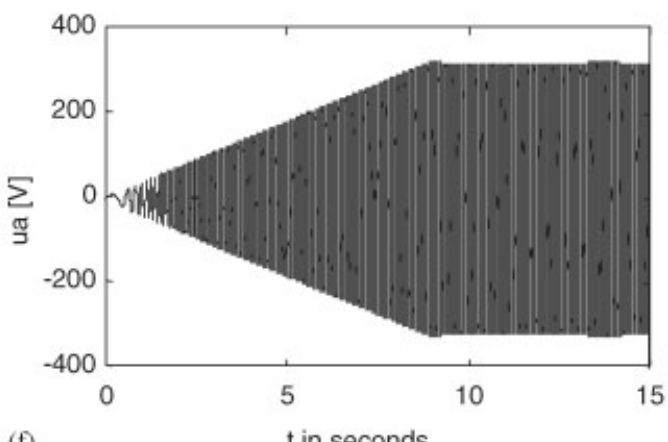

(f)

Figure 7. Results using MIMO_CFAG and MIMO_CTVAG for variations of motor parameters.

well as MIMO_CTVAG guaranty good results for a wide range of variations of the proportional gains.

\section{CONCLUSIONS}

Based on MIMO passivity concepts [25,26] and the principle called 'Principle of Torque-Flux Control' proposed in Reference [5], two simplified control schemes have been obtained. The 


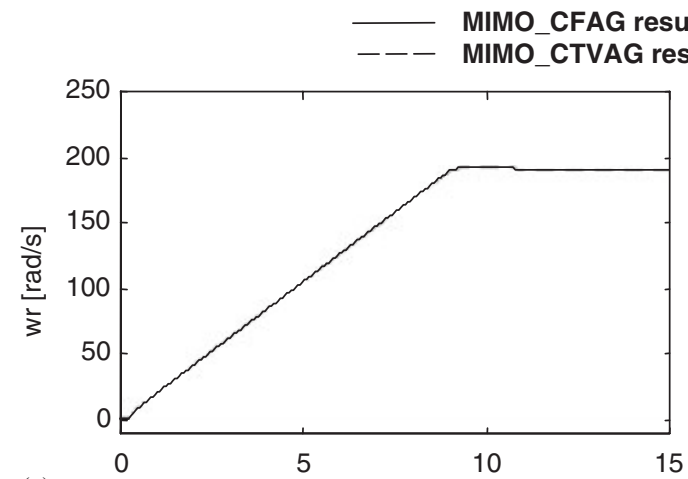

(a)
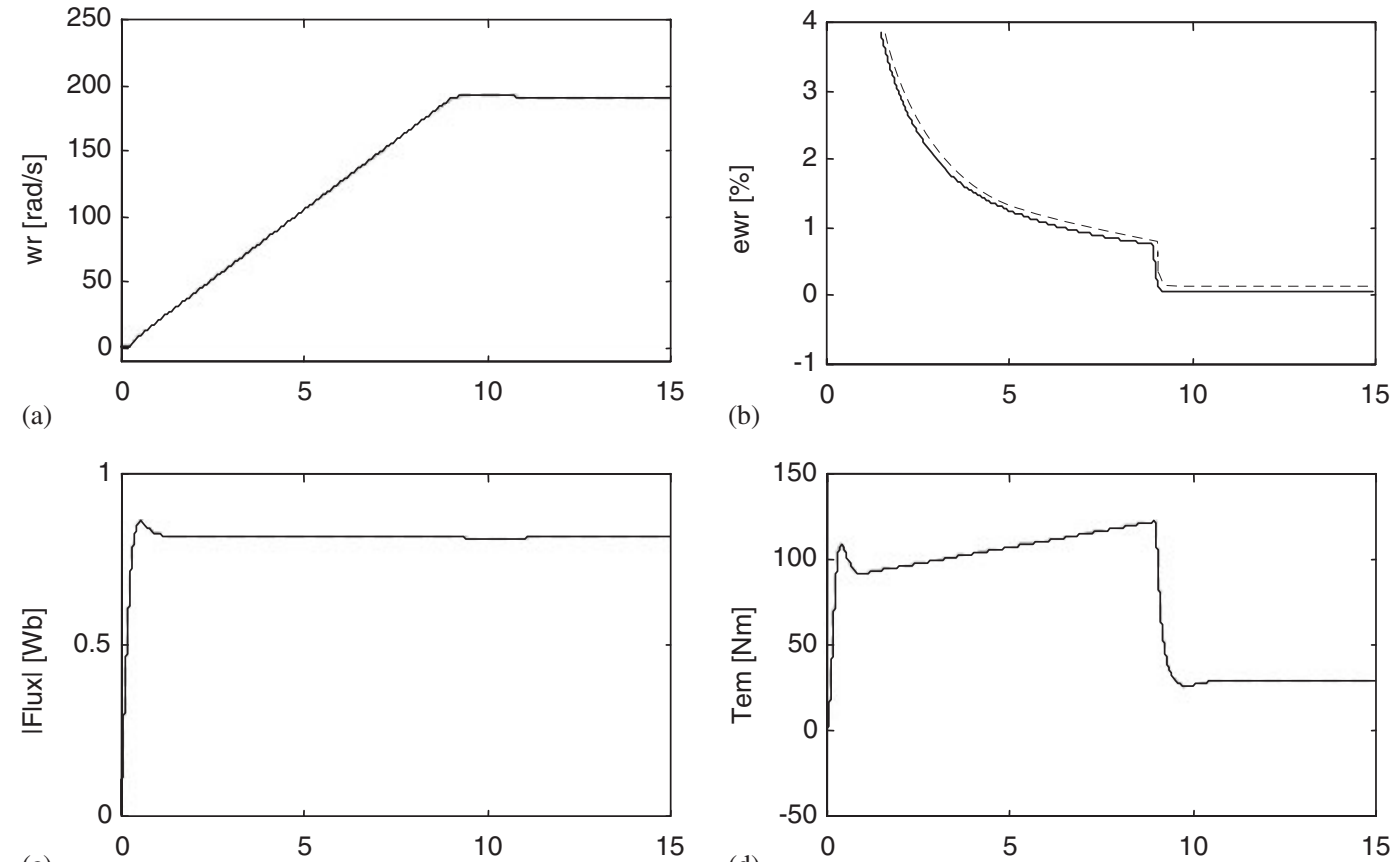

(c)
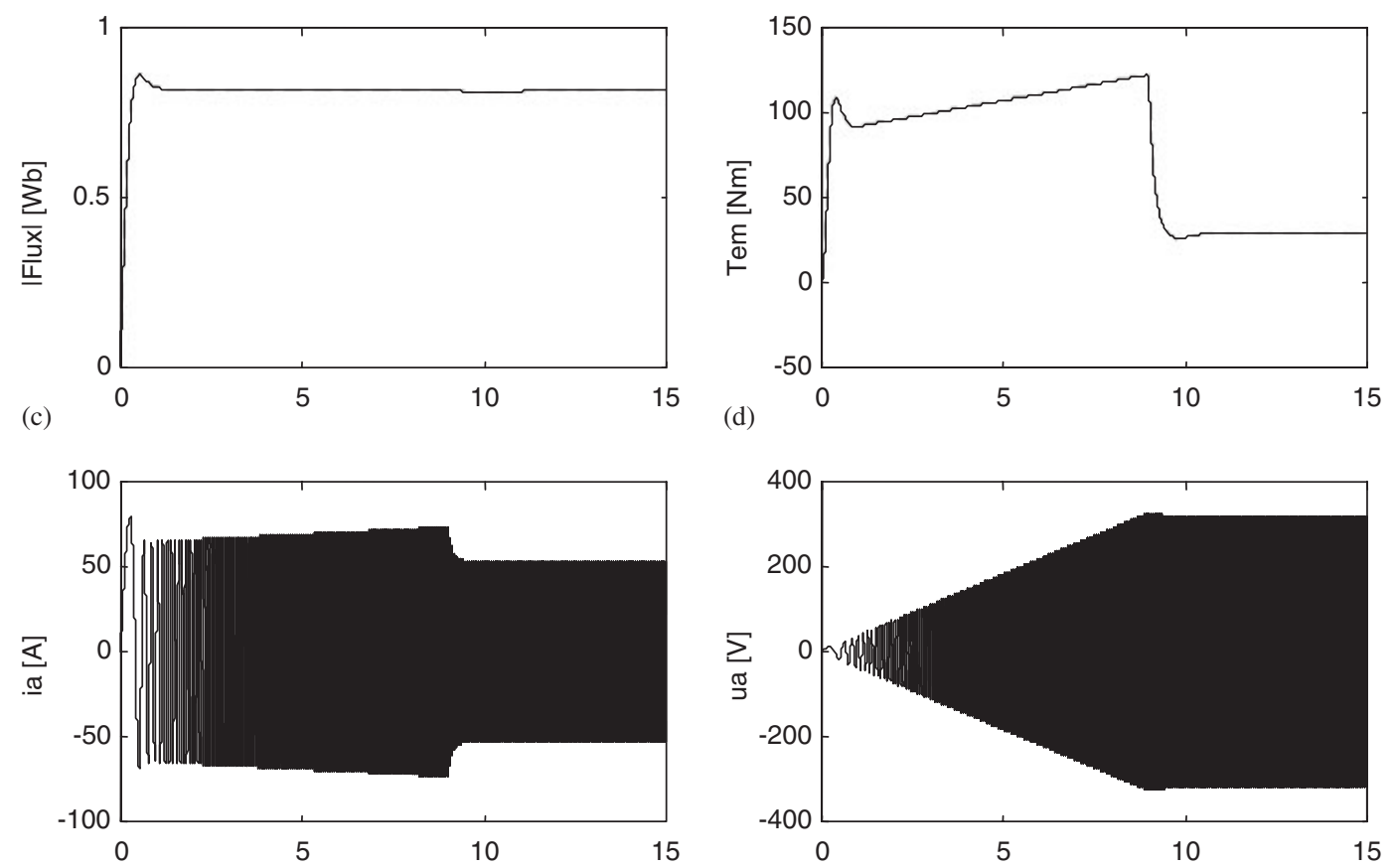

(e)

$\mathrm{t}$ in seconds

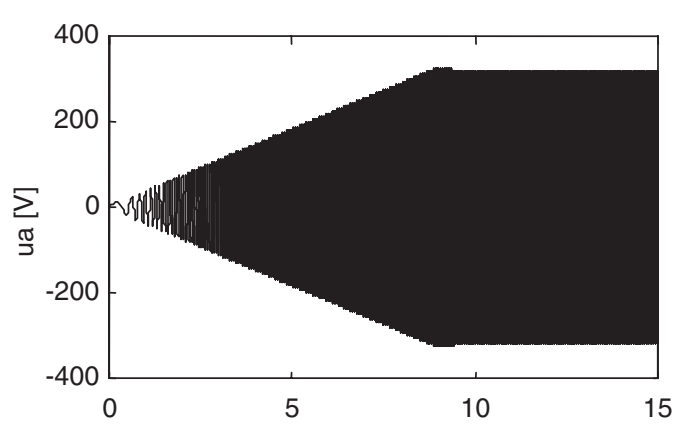

(f)

$\mathrm{t}$ in seconds

Figure 8. Results of MIMO_CFAG and MIMO_CTVAG under for variations of load parameters.

proposed controllers do not use state estimation (estimation or measurement of rotor flux or rotor currents) and no parameter estimation (parameters of the motor-load system), except those used for the field orientation block, are needed. One scheme considers constant adaptive gains for the adaptive laws whereas the other has time-varying adaptive gains, which allows an improvement in the transient behaviour of the controlled system.

Compared with other non-linear control schemes proposed in the literature such as sliding modes [1-4,10-22], we are in the presence of two simple and novel controllers. They have adaptive characteristics, are robust in the presence of load parameter variations and use simple 


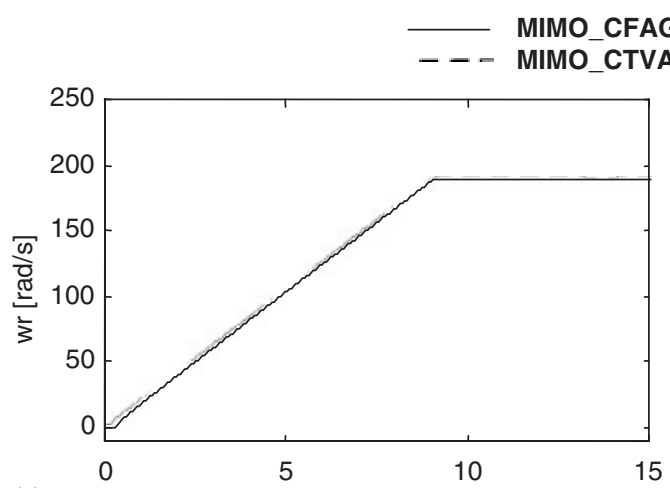

(a)

(b)
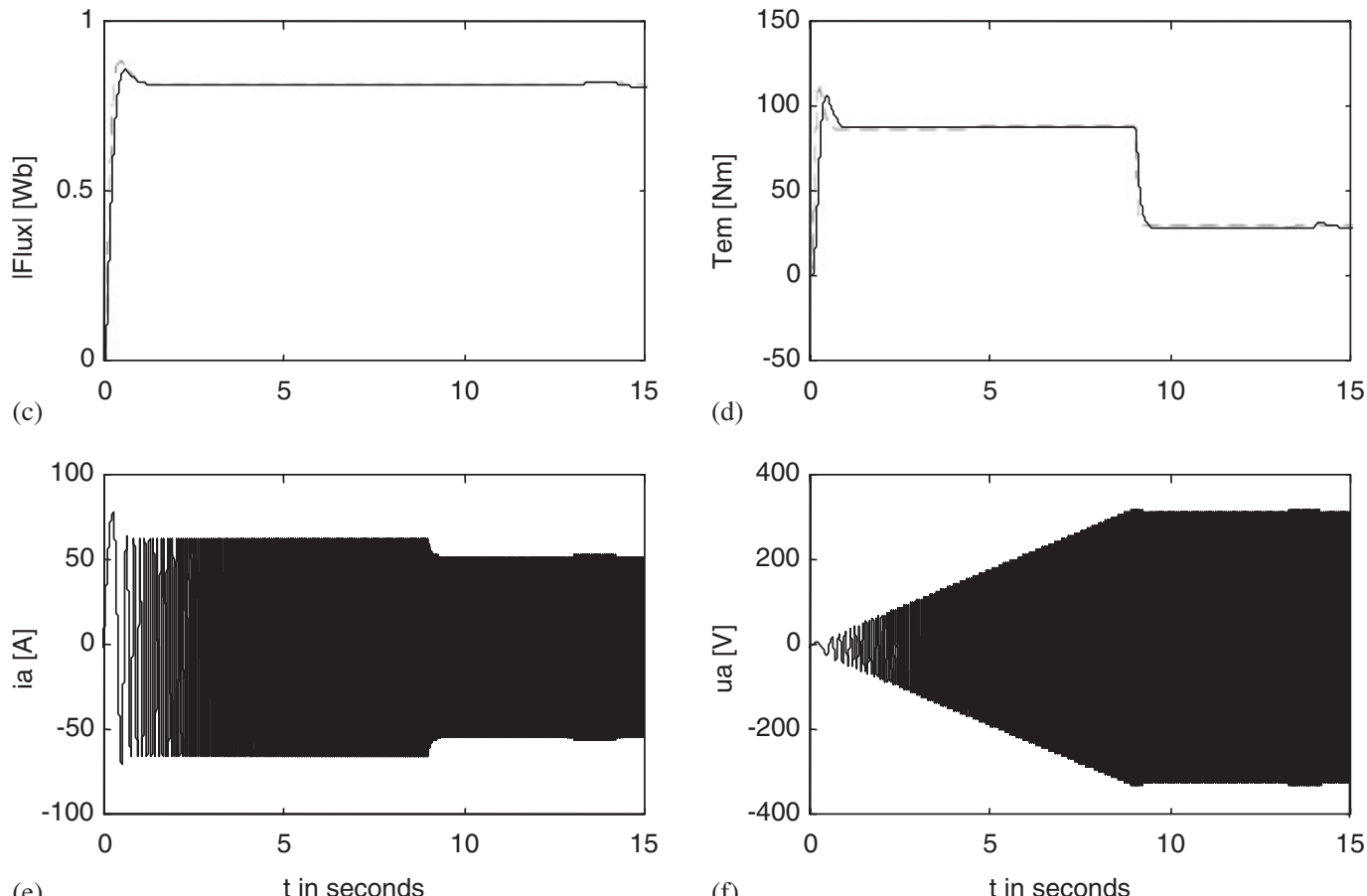

(d)

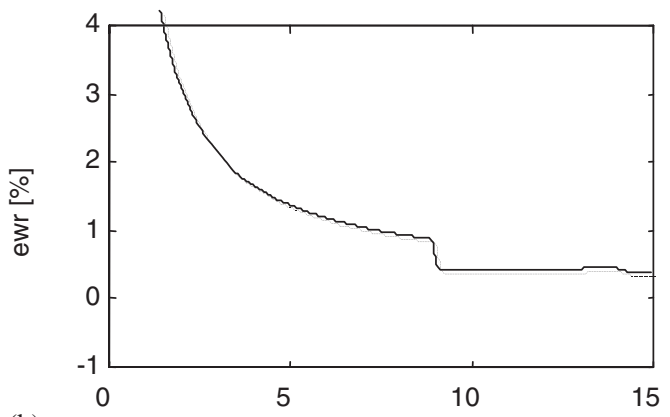

b)

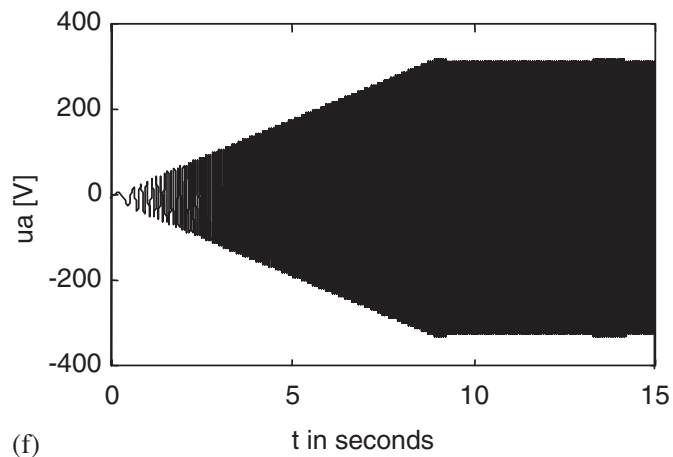

Figure 9. Results using MIMO_CFAG and MIMO_CTVAG under changes in the tuning of the proportional gains.

proportional controllers in the rotor speed, rotor flux and stator current control loops. They are also robust for a wide range of proportional gain variations.

Besides, the proposed control schemes guarantee high starting torque at low speed and during the transient, accuracy in steady state, wide range of speed control and good response under speed changes, obtaining a high performance control. The results obtained here are quite similar to those presented in Reference [5] for the SISO case, but from a MIMO perspective.

It was shown that the controller MIMO_CTVAG presents a slightly better transient response than the controller MIMO_CFAG, due to the time-varying adaptive gains included. Finally, the 
proposed controllers exhibit a much better behaviour than the classical scheme studied in Reference [5], which is based on the control scheme given in Reference [27].

\section{ACKNOWLEDGEMENTS}

The results reported in this paper have supported by CONICYT-CHILE through grant FONDECYT No 1000937. The support of Vicerrectoría de Investigación y Desarrollo, Universidad de Santiago de Chile (USACH), is greatly appreciated by Travieso.

\section{REFERENCES}

1. Chan CC, Wang HQ. New scheme of sliding-mode control for high performance induction motor drives. IEEE Proceedings on Electrical Power Applications 1996; 143(3):177-185.

2. Dunnigan MW, Wade S, Williams BW, Yu X. Position control of a vector controlled induction machine using Slotine's sliding mode control approach. IEE Proceedings on Electrical Power Applications 1998; 145:231-248.

3. Taoutaou D, Castro-Linares R. A controller-observer scheme for induction motors based on passivity feedback equivalence and sliding modes. International Journal of Adaptive Control and Signal Processing 2000; 14(2-3):355376.

4. Espinosa G, Ortega R. An output feedback globally stable controller for induction motors. IEEE Transaction on Automatic Control 1995; 40(1):138-143.

5. Travieso JC, Duarte MA. Two simple and novel siso controllers for induction motors based on adaptive passivity. IEEE Transaction on Industrial Electronics 2002, submitted.

6. Lorenz RD, Lipo TA, Novothy DW. Motion control with induction motors. Proceedings of the IEEE 1994; 82(8): $1215-1240$.

7. Bose BK (ed.). Power Electronics and Variable Frecuency Drives. Technology and applications. IEEE PRESS: New York, 1997.

8. Vas P. Artificial-Intelligence-Based Electrical Machines and Drives. Oxford University Press: Oxford, 1999.

9. Vas P. Sensorless vector and direct torque control. Oxford University Press: New York, 1998.

10. Benchaib A, Edwards C. Nonlinear sliding mode control of an induction motor. International Journal of Adaptive Control and Signal Processing 2000; 14(2-3):201-221.

11. Zheng Y, Abdel Fattah HA, Loparo KA. Non-linear adaptive sliding modes observer-controller sacheme for induction motors. International Journal of Adaptive Control and Signal Processing 2000; 14(2-3):245-273.

12. Araújo RE, Freitas DS. Non-linear control of induction motor: sliding mode theory loeads to robust and simple solution. International Journal of Adaptive Control and Signal Processing 2000; 14(2-3):331-353.

13. Aquino P, Feemster M, Dawson DM, Behal A. Adaptive partial state feedback control of the induction motor elimination of rotor flux and rotor velocity measurements. International Journal of Adaptive Control and Signal Processing 2000; 14(2-3):83-108.

14. Lin YC, Fu LC, Tsai CY. Non-linear sensorless indirect adaptive speed control of induction motor with unknown rotor resistance and load. International Journal of Adaptive Control and Signal Processing 2000; 14(2-3):109-140.

15. Martin $\mathrm{Ph}$, Rouchon P. Two simple flux observers for induction motors. International Journal of Adaptive Control and Signal Processing 2000; 14(2-3):171-175.

16. Damiano A, Gatto G, Marongiu I. An adaptive rotor flux observer for indirect field oriented induction motor drives. International Journal of Adaptive Control and Signal Processing 2000; 14(2-3):275-296.

17. Shishkin SL, Wunsch DC. Discrete-time method for robust glogal stabilization of induction motor. International Journal of Adaptive Control and Signal Processing 2000; 14(2-3):141-156.

18. Aloliwi B, Khalil HK, Strangas EG. Robust Speed Control of induction motors: application to a benchmark example. International Journal of Adaptive Control and Signal Processing 2000; 14(2-3):157-170.

19. Peresada S, Tonielli A. High-performance robust speed-flux tracking controller for induction motor. International Journal of Adaptive Control and Signal Processing 2000; 14(2-3):177-200.

20. Ding G, Wan X, Han Z. H- $\infty$ disturbance attenueation control of induction motor. International Journal of Adaptive Control and Signal Processing 2000; 14(2-3):223-244.

21. Maaziz MK, Boucher P, Dumur D. A new control strategy for induction motor based on non-linear predictive control and feedback linearization. International Journal of Adaptive Control and Signal Processing 2000; 14(2-3):313-329.

22. Lee HT, Chang JS, Fu LC. Exponential stable non-linear control for speed regulaton of induction motor with field oriented PI controller. International Journal of Adaptive Control and Signal Processing 2000; 14(2-3):297-312.

23. Castro R, Duarte M. Passivity equivalence of a class of SISO nonlinear systems via adaptive feedback. Proceedings of VIII Latinamerican Congress on Automatic Control, Marbella, Chile, November 9-13, 1998; 249-254. 
24. Duarte M, Castro R, Castillo A. Adaptive passivity of nonlinear systems using time-varying gains. Dynamics and Control 2001; 11(4):333-351.

25. Duarte M, Castro R, Castillo A. Direct passivity of a class of MIMO nonlinear systems using adaptive feedback. International Journal of Control 2002; 75(1):23-33.

26. Duarte M, Méndez J, Castro R, Castillo A. Adaptive passivation with time-varying gains of MIMO nonlinear systems. Kybernetes 2003, in press.

27. Chee-Mun O. Dynamic Simulation of Electric Machinery, Using Matlab/Simulink. Prentice-Hall: PTR, 1998.

28. Byrnes CI, Isidori A, Willems JC. Passivity, feedback equivalence and the global stabilization of minimum phase nonlinear systems. IEEE Transactions on Automatic Control 1991; 36(11):1228-1240. 